Supporting Information

Facile Fabrication of Polymer Electrolytes via Lithium Salt-Accelerated Thiol-Michael Addition for Lithium-Ion Batteries

Ke Jiang, Jirong Wang, Cai Zuo, Shaoqiao Li, Sibo Li, Dan He, Haiyan Peng, Xiaolin Xie, Rinaldo Poli, * and Zhigang Xue*

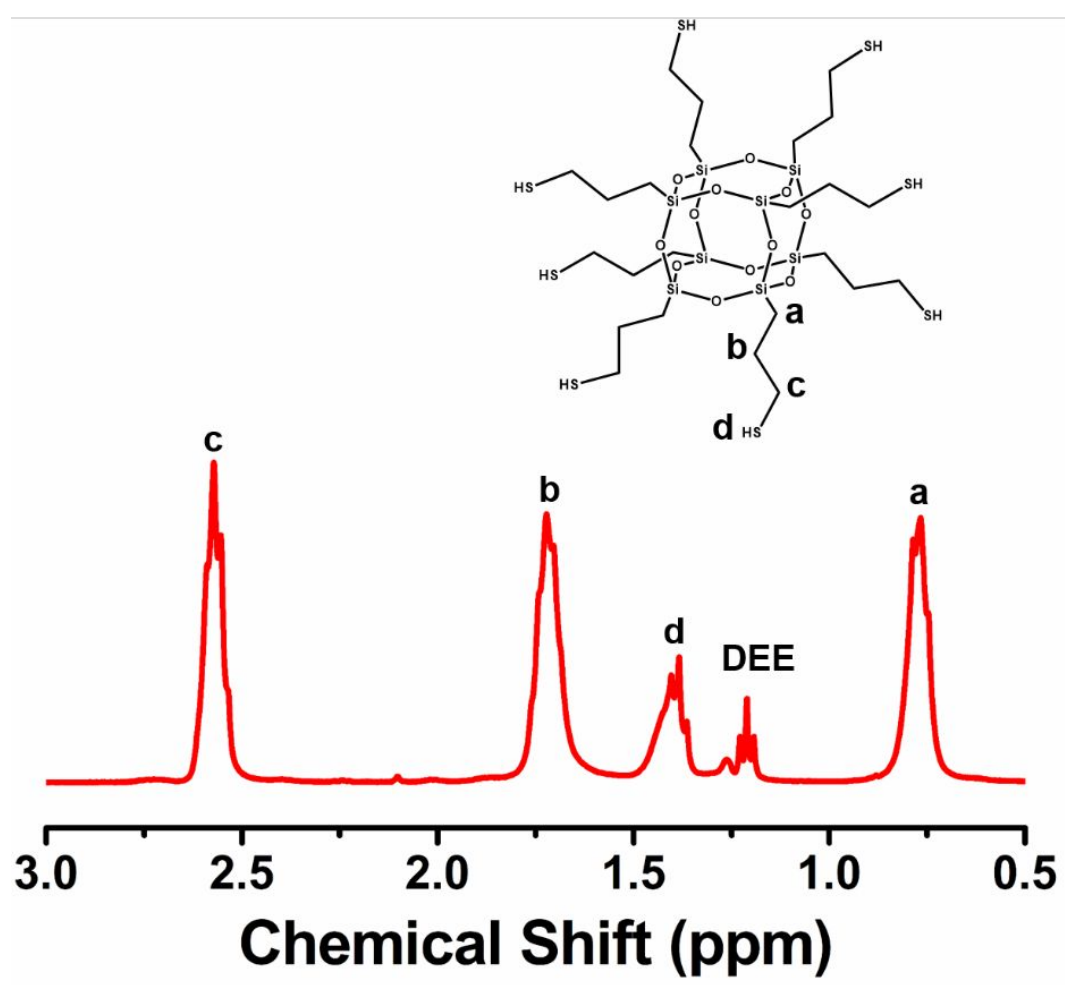

Figure S1. ${ }^{1} \mathrm{H}$ NMR spectrum $\left(\mathrm{CDCl}_{3}\right)$ of synthesized POSS-SH.

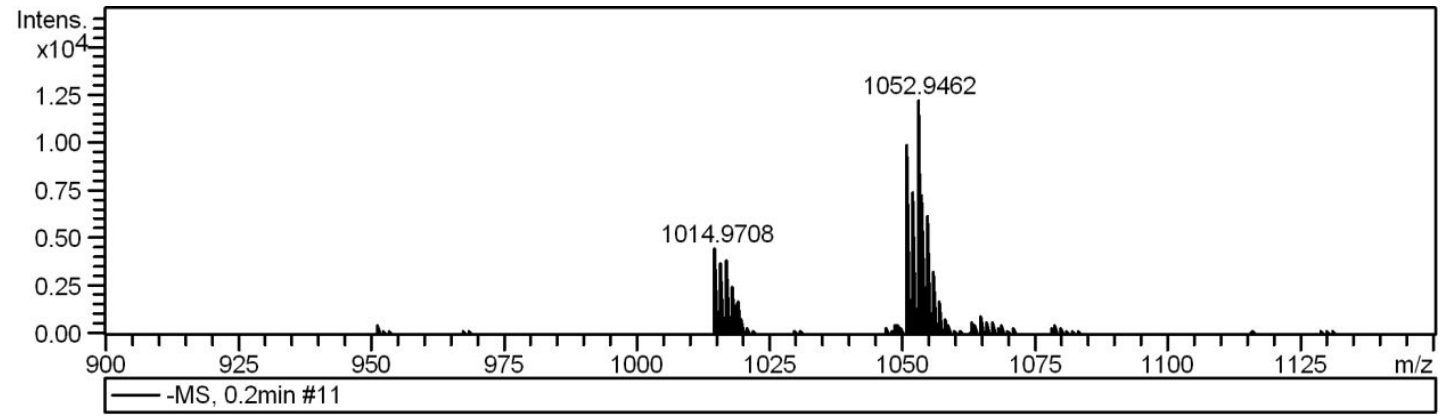

Figure S2. Mass spectrum of POSS-SH. 


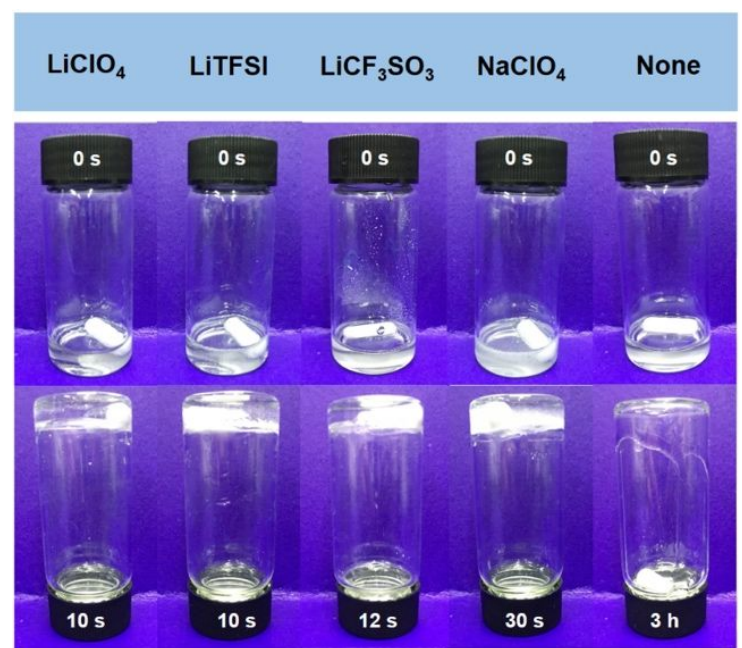

Figure S3. Images of PEGDA 600 and PETMP mixed with alkali metal salts, and cross-linking polymer electrolytes after stored at room temperature.

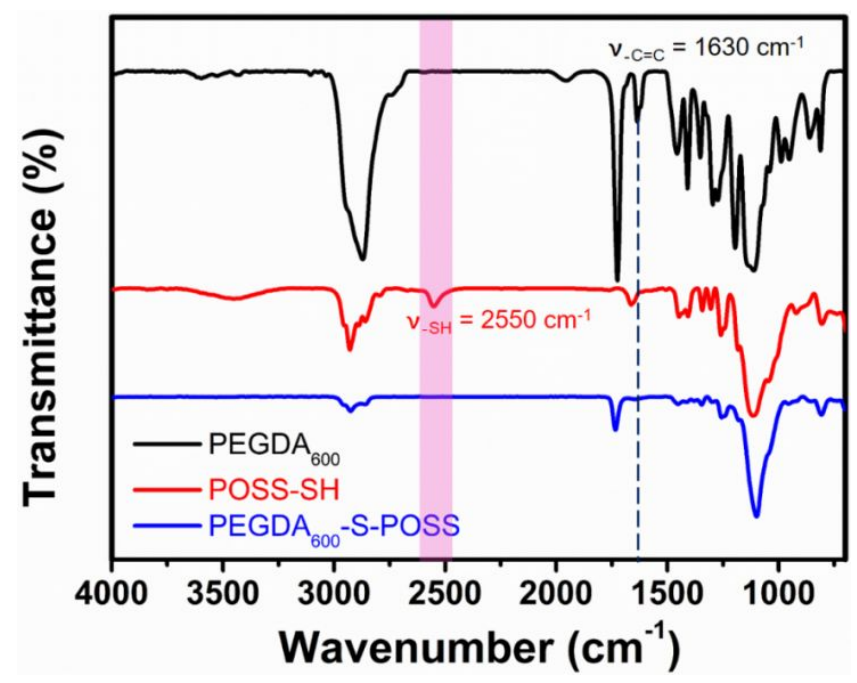

Figure S4. FT-IR spectra of PEGDA 600 , POSS-SH, and PEGDA $600-\mathrm{S}-\mathrm{POSS}$.

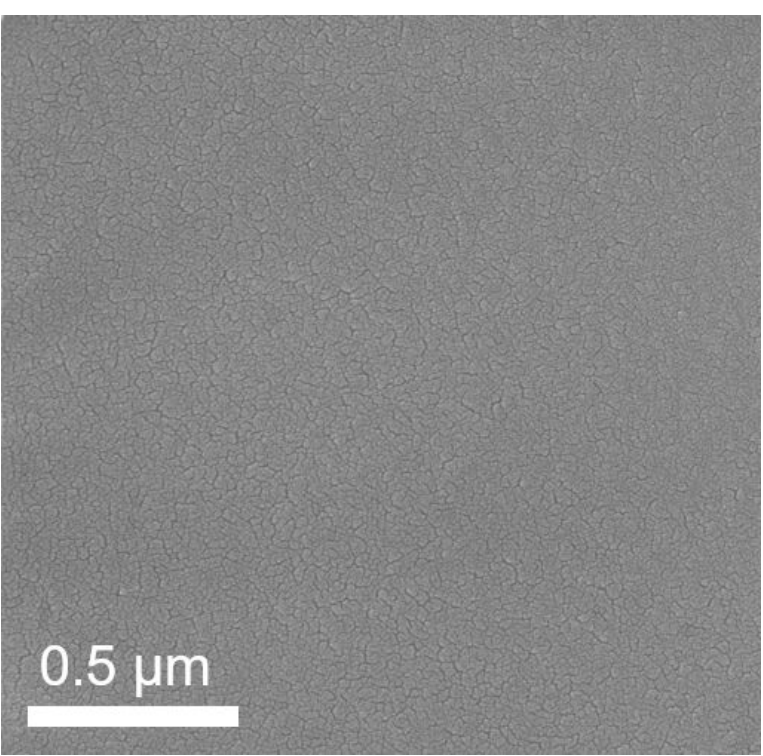

Figure S5. SEM image of PEGDA600-S-POSS. 

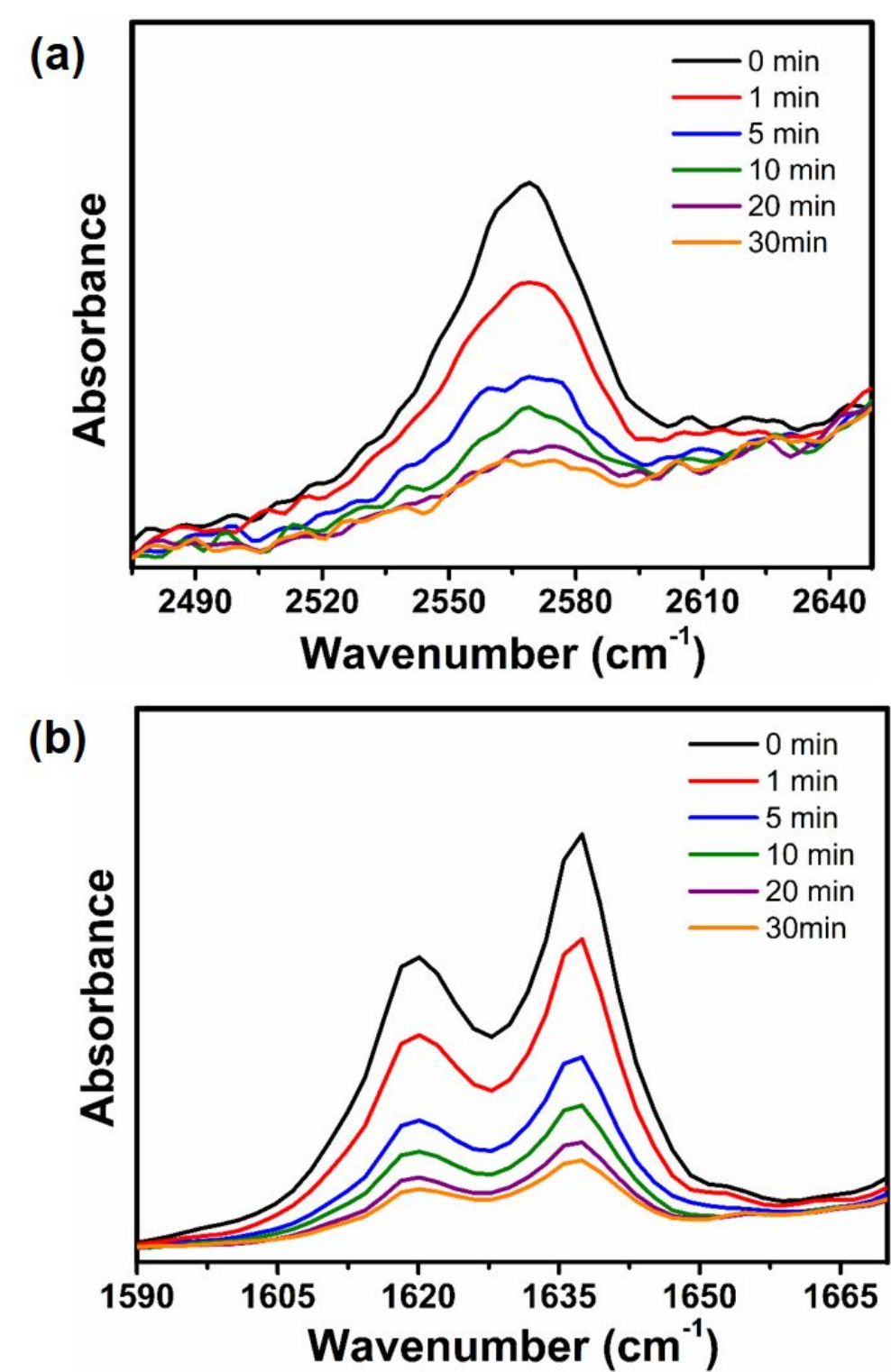

Figure S6. FT-IR spectra of (a) thiol and (b) acrylate peaks for the TEA-catalyzed thiolMichael addition of PEGDA 200 and 1-hexanethiol (HT). [HT] : [PEGDA 200$]$ : [TEA] = 2:1:0.043 (thiol : vinyl $=1: 1$ ) with PEGDA $200: \mathrm{LiClO}_{4}=20: 1$.
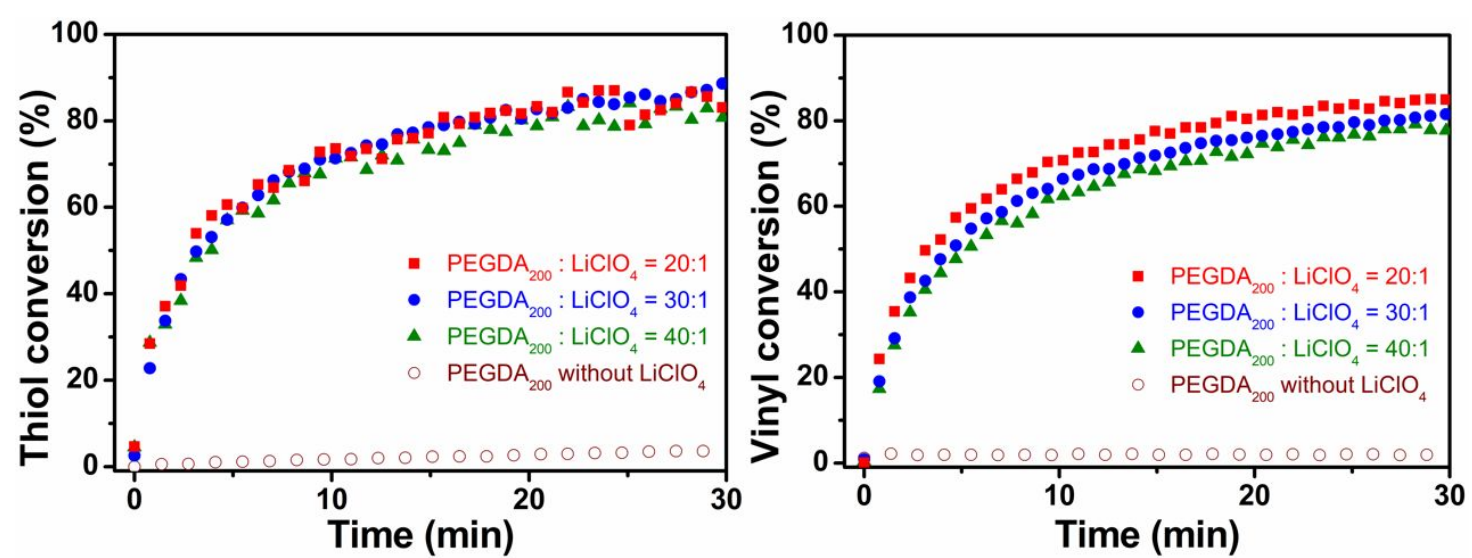

Figure S7. Conversion against time of the thiol functional group and vinyl group for the TEAcatalyzed thiol-Michael addition of PEGDA $_{200}$ and 1-hexanethiol (HT). [HT] : [PEGDA 200$]$ : $[\mathrm{TEA}]=2: 1: 0.043$ (thiol : vinyl $=1: 1$ ) in the presence of different ratio of $\mathrm{LiClO}_{4}$. 


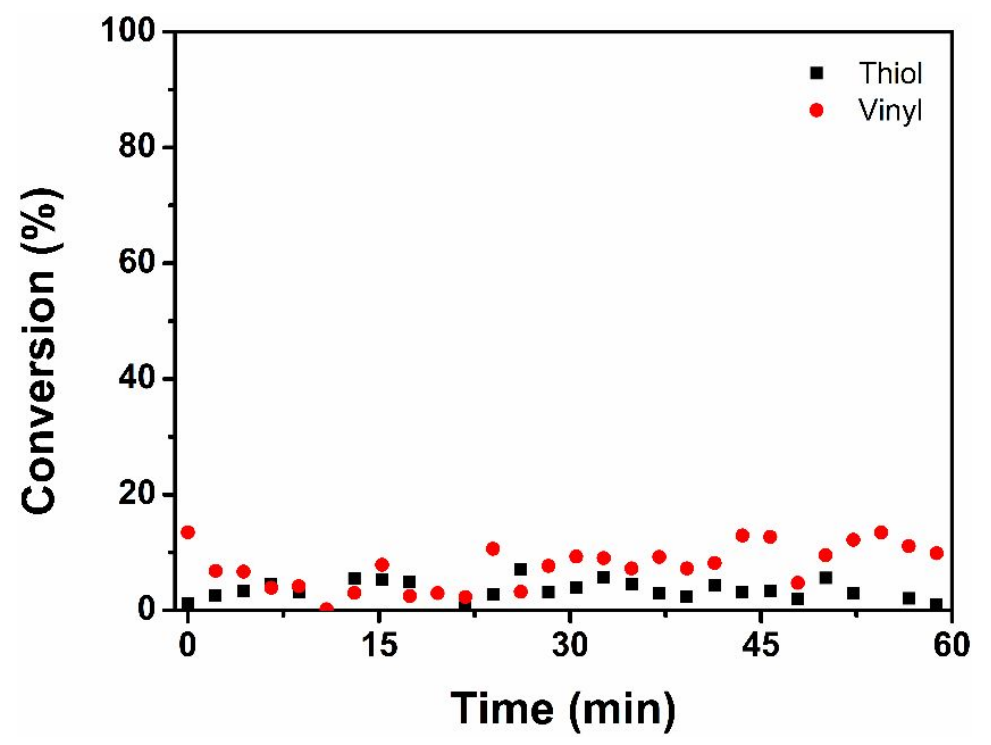

Figure S8. Conversion against time for $[\mathrm{HT}]:\left[\mathrm{PEGDA}_{200}\right]=2: 1($ thiol $:$ vinyl $=1: 1)$ with $\mathrm{LiClO}_{4}$ only.

(a)

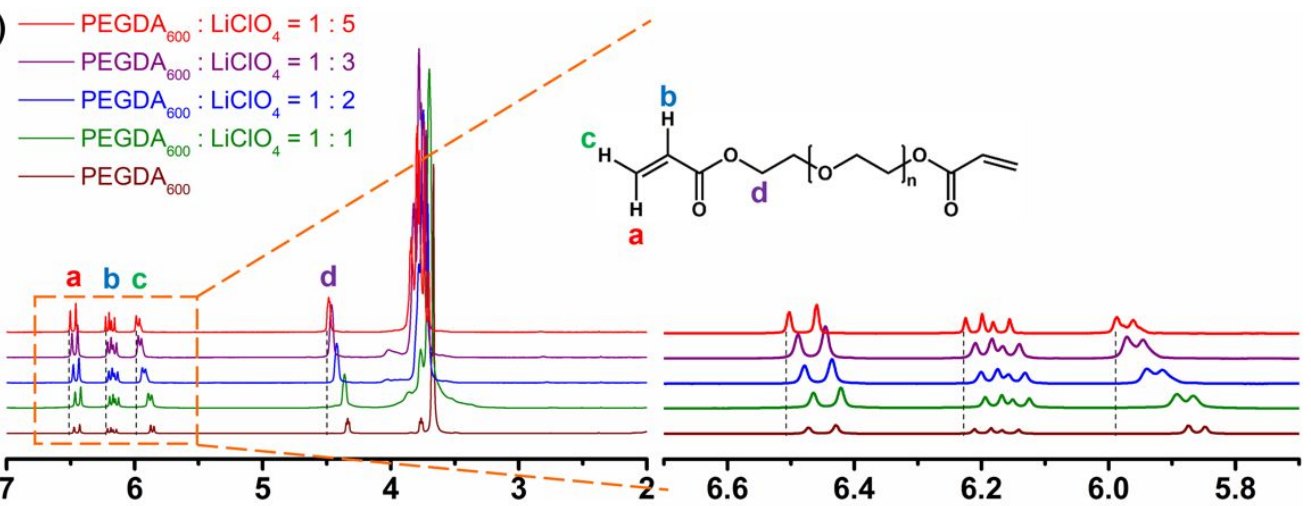
(b) - $\mathrm{PEGDA}_{600}: \mathrm{LiClO}_{4}=1: 2$
PEGDA ${ }_{600}:$ LITFSI $^{2}=1: 2$
$-\mathrm{PEGDA}_{600}: \mathrm{LiCF}_{3} \mathrm{SO}_{3}=1: 2$
$-\mathrm{PEGDA}_{600}: \mathrm{NaClO}_{4}=1: 2$
- PEGDA $600: \mathrm{KClO}_{4}=1: 2$
- PEGDA ${ }_{600}$
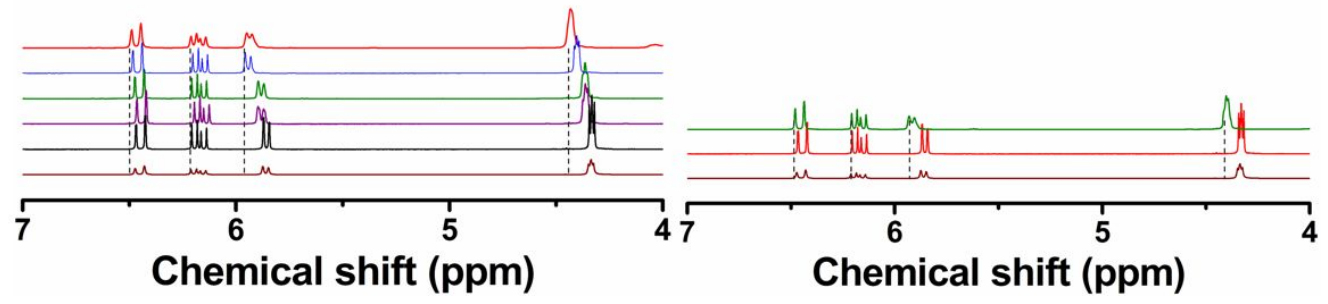

Figure S9. (a) ${ }^{1} \mathrm{H}$ NMR spectra $\left(\mathrm{CDCl}_{3}\right)$ of $\mathrm{PEGDA}_{600}$ with different ratios of $\mathrm{LiClO}_{4}$ and without $\mathrm{LiClO}_{4}$. The spectra with selected chemical shift region between 7.0 and $5.2 \mathrm{ppm}$ are illustrated concomitantly. (b) ${ }^{1} \mathrm{H}$ NMR spectra $\left(\mathrm{CDCl}_{3}\right)$ of $\mathrm{PEGDA}_{600}$ with different alkali metal salts. (c) ${ }^{1} \mathrm{H}$ NMR spectra $\left(\mathrm{CDCl}_{3}\right)$ of $\mathrm{PEGDA}_{600}$ with TEA and $\mathrm{LiClO}_{4}$. 
Table S1. Relevant DFT-optimized parameters and calculated physical parameters for the $\mathrm{MA} \cdots \mathrm{MtClO}_{4}$ molecules in $\mathrm{CHCl}_{3}$.

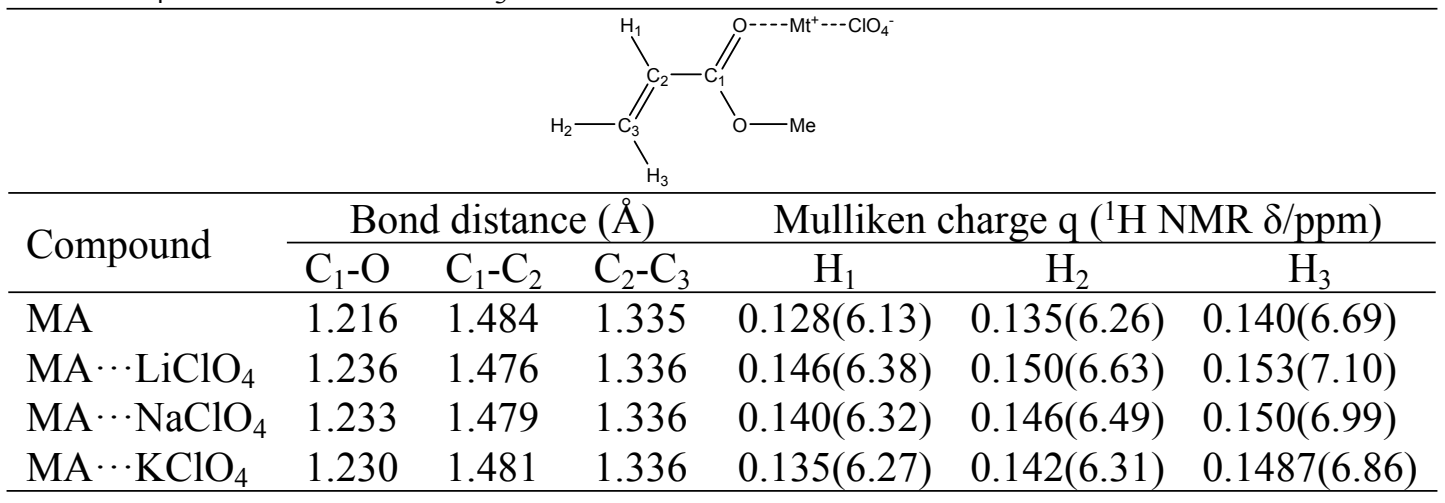

(a)<smiles>[R]OC(=O)/C=C\CCO</smiles>

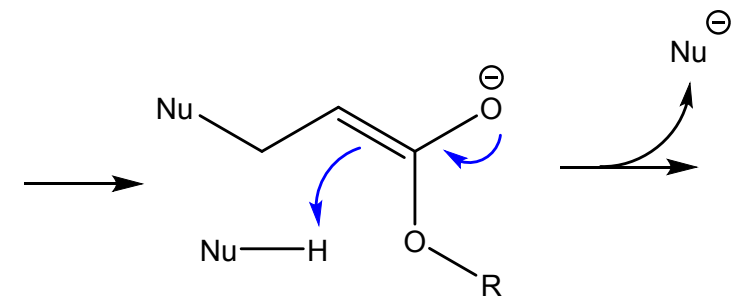<smiles>[R]OC(=O)CCN</smiles>

(b)

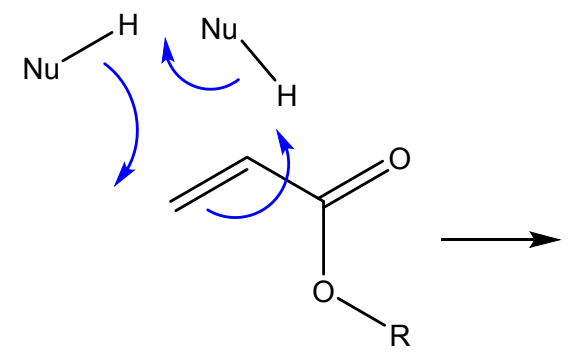

(c)<smiles>[R]OC(=O)/C=C\CCCO</smiles>

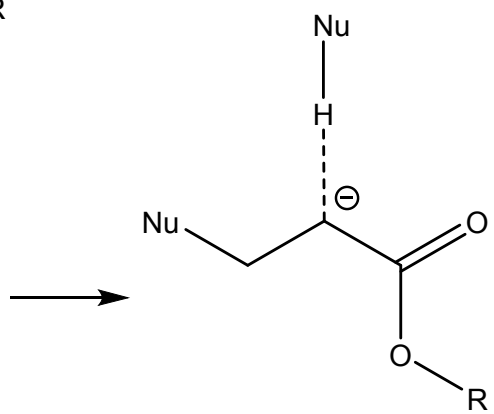<smiles>CC1CC1[N+](=O)[O-]</smiles><smiles>[R]OC(=O)CCN</smiles>

Scheme S1. Mechanism of the thiol-Michael addition to acrylate: (a) traditional (stepwise) mechanism; (b) concerted nucleophile and proton addition, which is followed by neutral thiol (c) alternative stepwise mechanism, which is followed by the anionic thiolate (free or associated to a cation). 


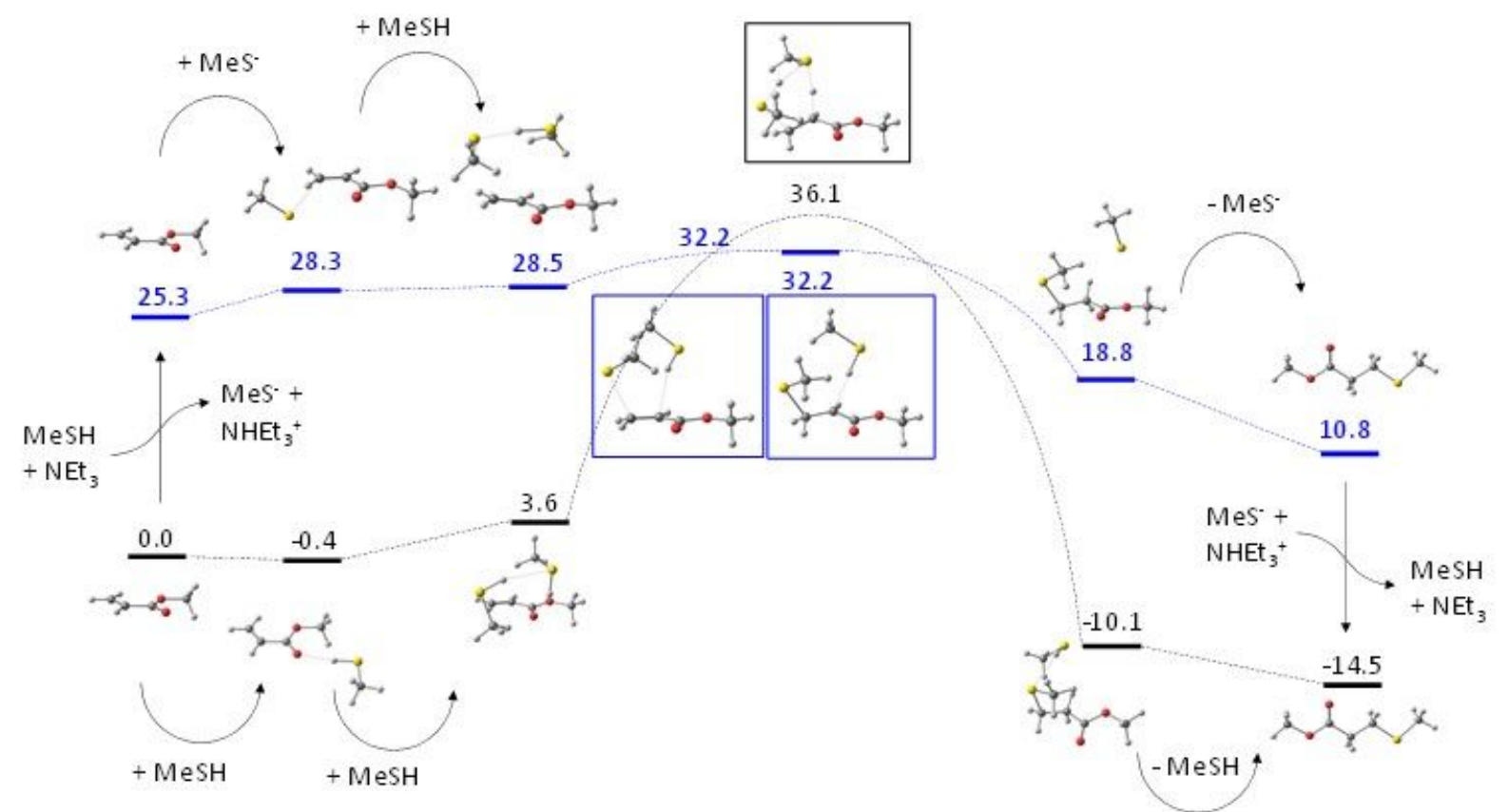

Figure S10. DFT-computed free energy profile $\left(\Delta G_{\mathrm{D} 3,298 \mathrm{~K}, \mathrm{THF}}\right.$ in $\left.\mathrm{kcal} / \mathrm{mol}\right)$ for the thiolMichael addition to methyl acrylate for the neutral and anionic nucleophile.

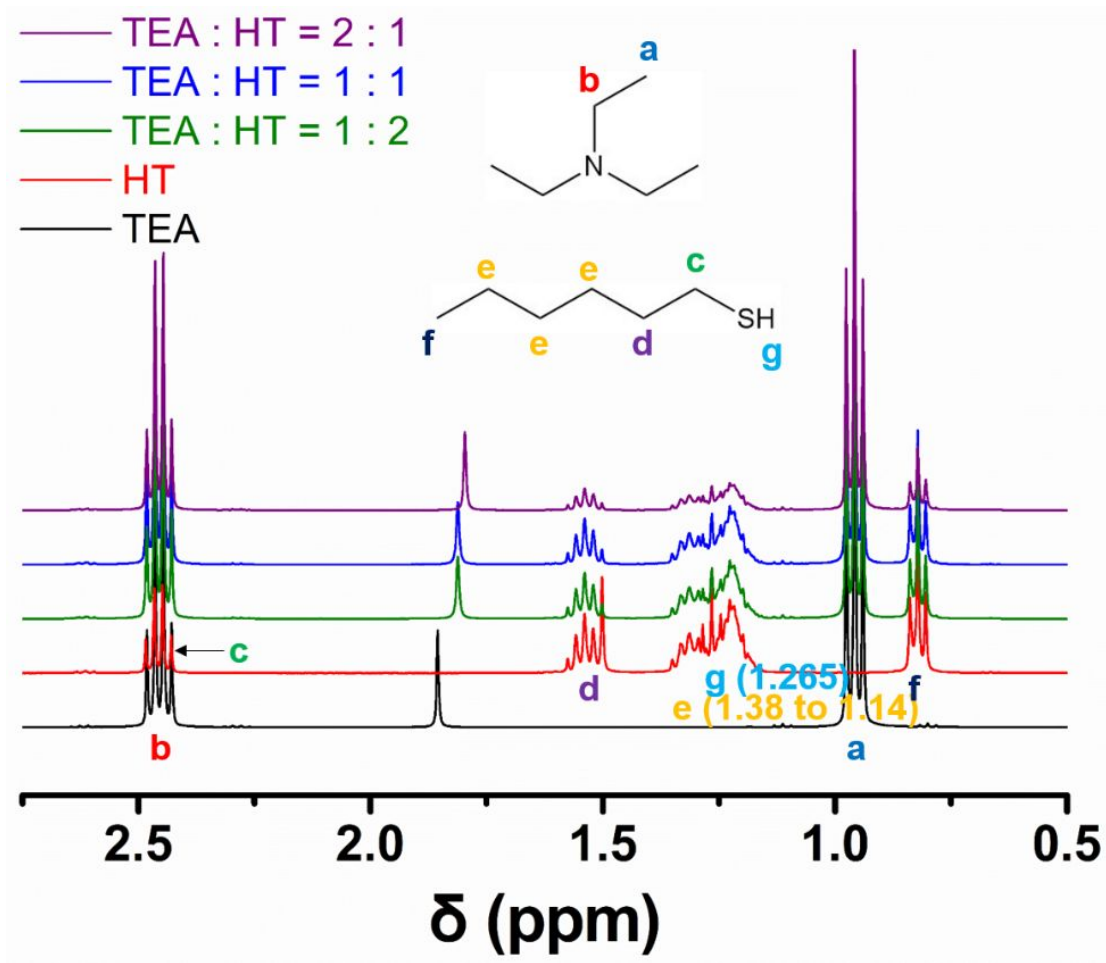

Figure S11. ${ }^{1} \mathrm{H}-\mathrm{NMR}$ study in $\mathrm{CDCl}_{3}$ of the interaction between $\mathrm{NEt}_{3}$ (TEA) and 1-hexanethiol (HT) at various ratios and comparison with the spectra of free TEA and HT. 


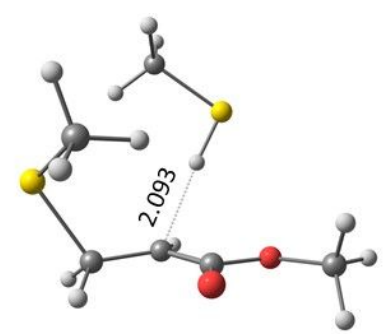

$\left[\mathrm{MeS}-\mathrm{CH}_{2} \mathrm{CH}(\mathrm{COOMe})\right]^{-} . . . \mathrm{HSMe}$

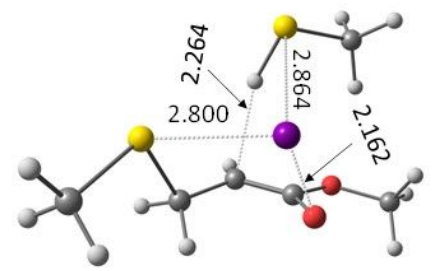

$\left[\mathrm{MeS}-\mathrm{CH}_{2} \mathrm{CH}(\mathrm{COOMe})\right]^{-} . . . \mathrm{Na}^{+}(\mathrm{HSMe})$

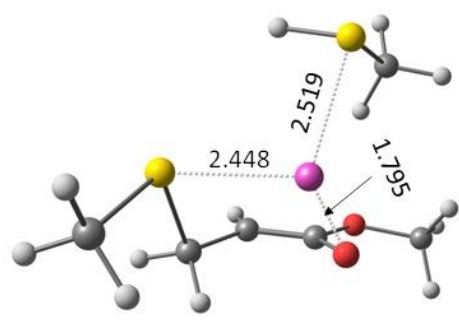

$\left[\mathrm{MeS}-\mathrm{CH}_{2} \mathrm{CH}(\mathrm{COOMe})\right]^{-} \ldots \mathrm{Li}^{+}(\mathrm{HSMe})$

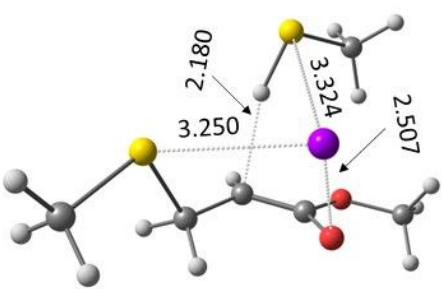

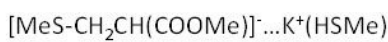

Figure S12. Views and selected interatomic distances for the optimized intermediates of the anionic pathway for the thiol-Michael addition.

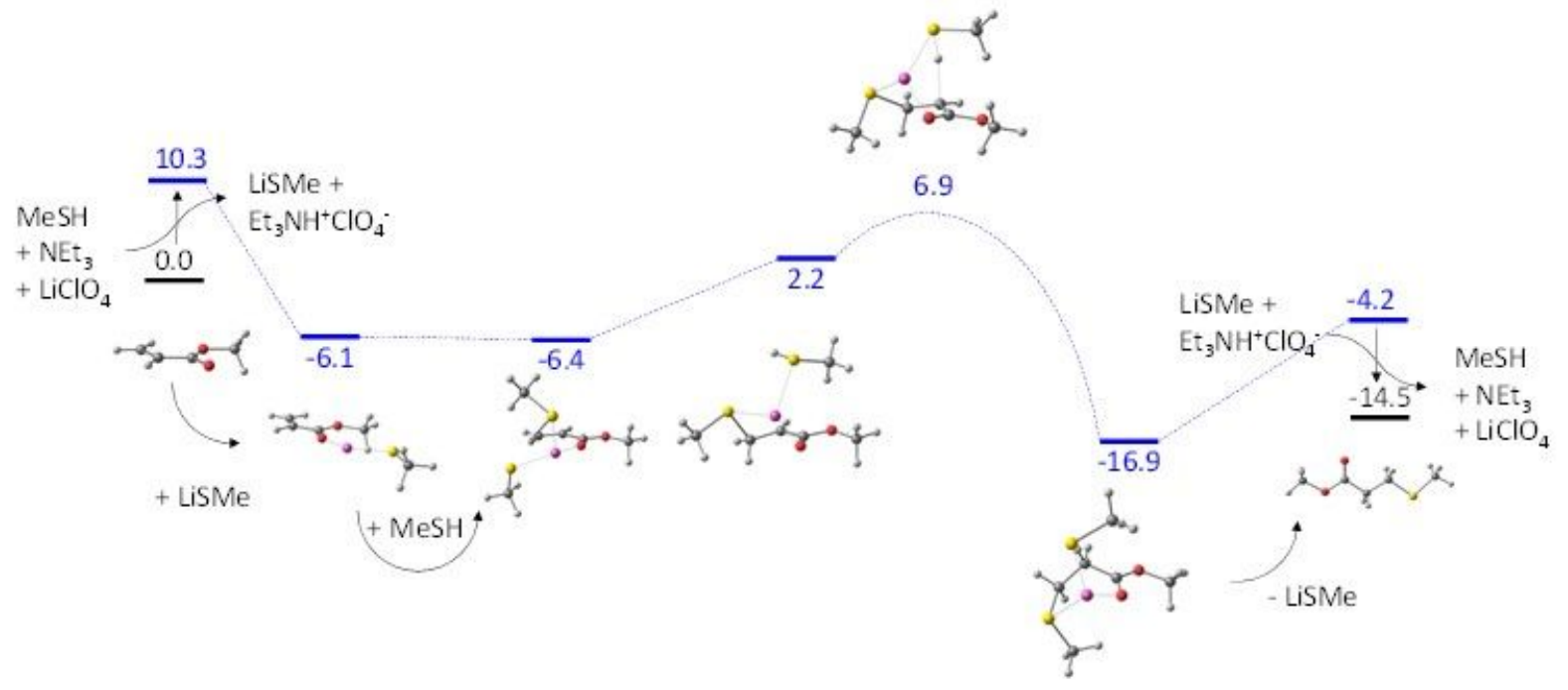

Figure S13. DFT-computed free energy profile $\left(\Delta G_{\mathrm{D} 3,298 \mathrm{~K}, \mathrm{THF}}\right.$ in $\left.\mathrm{kcal} / \mathrm{mol}\right)$ for the thiolMichael addition to methyl acrylate for Li salt of the anionic nucleophile.

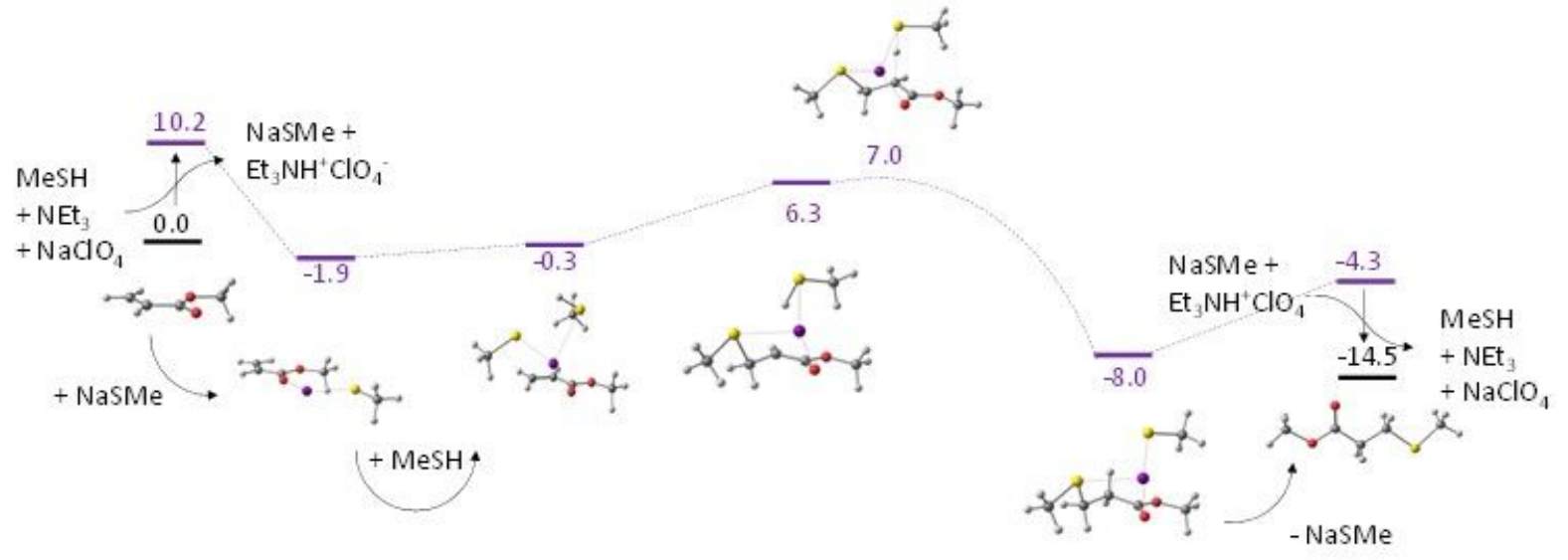

Figure S14. DFT-computed free energy profile $\left(\Delta G_{\mathrm{D} 3,298 \mathrm{~K}, \mathrm{THF}}\right.$ in $\left.\mathrm{kcal} / \mathrm{mol}\right)$ for the thiolMichael addition to methyl acrylate for Na salt of the anionic nucleophile. 


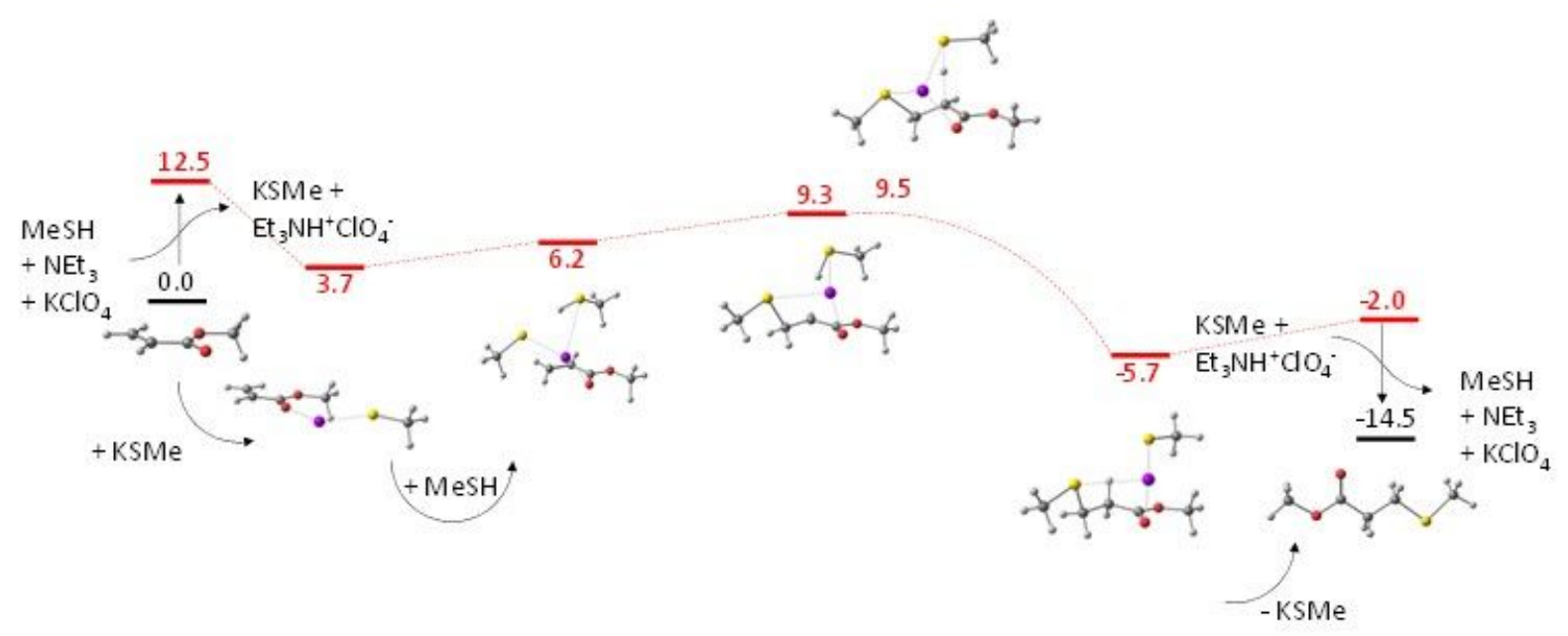

Figure S15. DFT-computed free energy profile $\left(\Delta G_{\mathrm{D} 3,298 \mathrm{~K}, \mathrm{THF}}\right.$ in $\left.\mathrm{kcal} / \mathrm{mol}\right)$ for the thiolMichael addition to methyl acrylate for $\mathrm{K}$ salt of the anionic nucleophile.

Table S2. Cartesian coordinates, views, and energies (hartrees) for all calculated systems.

\section{Starting materials}

Methyl acrylate (MA), $\mathrm{CH}_{2}=\mathrm{CH}-\mathrm{COOCH}_{3}$ $E=-306.469655708$

$E-D 3=-306.476209941$

$E_{\text {CHCI3 }}=-306.468816584$

$E_{\text {THF }}=-\mathbf{3 0 6 . 4 7 0 0 5 7 3 2 5}$

$\mathrm{G}_{\text {corr.,298K, gas }}=\mathbf{0 . 0 6 5 1 5 7}$

$\begin{array}{lr}6 & 0.010892000 \\ 6 & 1.491018000 \\ 8 & -0.590213000 \\ 8 & -0.603471000 \\ 6 & -2.037704000 \\ 1 & -2.406717000 \\ 1 & -2.370426000 \\ 1 & -2.406866000 \\ 1 & 1.987798000 \\ 6 & 2.173063000 \\ 1 & 1.663413000 \\ 1 & 3.258659000\end{array}$

Methyl thiol, $\mathrm{CH}_{5} \mathrm{SH}$

$E=-438.702078307$

$E-D 3=-438.703263389$

$E_{T H F}=-438.70694297$

$\mathrm{G}_{\text {corr.,298k,gas }}=0.021994$

$\begin{array}{lr}16 & -0.668296000 \\ 6 & 1.166222000 \\ 1 & 1.536158000 \\ 1 & 1.536141000 \\ 1 & 1.531845000 \\ 1 & -0.908742000\end{array}$

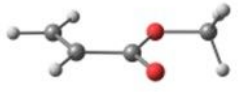

0.484636000

0.384012000

1.541407000

$-0.721775000$

$-0.671638000$

$-0.149377000$

$-1.709766000$

$-0.151086000$

1.349212000

$-0.763881000$

$-1.721598000$

$-0.773219000$

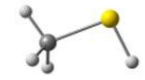

$-0.087035000$

0.019440000

0.522774000

0.522846000

$-1.009434000$

1.239738000
0.000110000

0.000026000

0.000143000

$-0.000010000$

$-0.000106000$

$-0.887283000$

$-0.001100000$

0.888025000

0.000083000

$-0.000108000$

$-0.000163000$

$-0.000160000$

MA $\cdots M e S H \cdot \cdots M e S H$

$E=\mathbf{- 1 1 8 3 . 8 8 2 6 7 7 2 9}$

$E-D 3=-1183.90716553$

$E_{T H F}=-1183.89285547$

$\mathrm{G}_{\mathrm{corr}, 298 \mathrm{~K}, \mathrm{gas}}=0.145329$

$-2.441347000$

1.557138000

1.298633000

0.765686000

2.802494000

3.154972000

3.001216000

4.207645000

2.547716000

2.143756000

$-3.063289000$

$-2.600407000$

$-3.035306000$

$-1.516010000$

$-3.019117000$
$-0.218838000$

$-0.308505000$

$-1.071334000$

$-0.847710000$

1.026443000

1.819080000

1.594763000

2.856357000

1.627129000

$-2.147292000$

$-0.515624000$

0.561883000

$-1.126262000$

0.410091000

$-1.091029000$

$-0.747151000$

$-1.723155000$

$-1.664272000$
0.030674000

0.007994000

0.007677000

0.027119000

$-0.014708000$

$-0.014905000$

$-0.893347000$

$-0.037509000$

0.886192000

0.026153000

$-0.012959000$

$-0.031310000$

$-0.011993000$

0.027159000

$-0.043077000$

$-0.051849000$

0.833762000

$-0.952982000$
$E=-745.176300654$

$E-D 3=-745.188008808$

$E_{\text {THF }}=-\mathbf{7 4 5 . 1 8 5 4 5 3 7 6 2}$

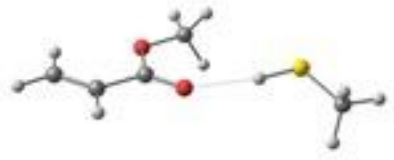

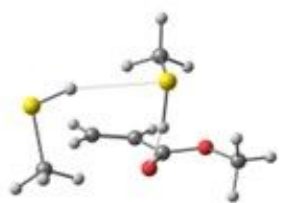

0.894161000

$-0.973470000$

$-2.047095000$

$-0.559910000$

$-0.475634000$

0.593894000

0.288584000

0.801094000

1.480086000

$-2.366793000$

$-0.305715000$

$-0.707781000$

$-1.685518000$

$-0.760623000$

0.024231000
$-0.281173000$

0.191092000

$-0.795299000$

1.023703000

0.047433000

0.946167000

1.984133000

0.755860000

0.741943000

$-1.396889000$

$-0.235498000$

1.499271000

1.718201000

1.606207000

2.192709000 


\begin{tabular}{|c|c|}
\hline \multicolumn{2}{|c|}{ MA $\cdots$ MeSH $\cdots$ MeSH TS } \\
\hline \multicolumn{2}{|c|}{$E=-1183.8301233$} \\
\hline \multicolumn{2}{|c|}{$E-D 3=-1183.85400657$} \\
\hline \multicolumn{2}{|c|}{$E_{T H F}=-1183.84242126$} \\
\hline \multicolumn{2}{|c|}{$G_{\text {corr.,298K,gas }}=0.146199$} \\
\hline 6 & 1.723832000 \\
\hline 6 & 0.561034000 \\
\hline 8 & 1.788401000 \\
\hline 8 & 2.721329000 \\
\hline 6 & 3.853093000 \\
\hline 1 & 4.330035000 \\
\hline 1 & 4.539117000 \\
\hline 1 & 3.550441000 \\
\hline 1 & 0.712908000 \\
\hline 6 & -0.309313000 \\
\hline 1 & 0.019163000 \\
\hline 1 & -0.850104000 \\
\hline 16 & -2.150609000 \\
\hline 1 & -1.877716000 \\
\hline 6 & -1.373169000 \\
\hline 1 & -1.522091000 \\
\hline 1 & -0.305612000 \\
\hline 1 & -1.861636000 \\
\hline 16 & -1.074145000 \\
\hline 1 & -0.102829000 \\
\hline 6 & -1.923630000 \\
\hline 1 & -1.198958000 \\
\hline 1 & -2.464683000 \\
\hline 1 & -2.640892000 \\
\hline
\end{tabular}

\section{MeS- $\mathrm{CH}_{2} \mathrm{CH}_{2}-\mathrm{COOCH}_{3} \cdots \mathrm{MeSH}$ \\ $\mathrm{E}=-\mathbf{1 1 8 3 . 9 0 7 3 3 7}$ \\ $E-D 3=-1183.93037911$ \\ $E_{\text {THF }}=-1183.92110325$ \\ $\mathrm{G}_{\text {corr.,298k,gas }}=0.150399$}

$\begin{array}{lr}6 & 1.834896000 \\ 6 & 0.447562000 \\ 8 & 2.732532000 \\ 8 & 1.962261000 \\ 6 & 3.264116000 \\ 1 & 4.026356000 \\ 1 & 3.195469000 \\ 1 & 3.520077000 \\ 1 & 0.515288000 \\ 6 & 0.001650000 \\ 1 & 0.841210000 \\ 1 & -0.807636000 \\ 16 & -0.701074000 \\ 1 & -2.088380000 \\ 6 & 0.689733000 \\ 1 & 1.612831000 \\ 1 & 0.837066000 \\ 1 & 0.420431000 \\ 16 & -2.786267000 \\ 1 & -0.262282000 \\ 6 & -3.399409000 \\ 1 & -2.575721000 \\ 1 & -3.977652000 \\ 1 & -4.049225000\end{array}$

$\mathrm{MeS}-\mathrm{CH}_{2} \mathrm{CH}_{2}-\mathrm{COOCH}_{3}$

$\begin{array}{cc}-2.559920000 & -0.944222000 \\ -2.196422000 & -0.353110000 \\ -3.334438000 & -1.677205000 \\ 2.481729000 & -0.084281000 \\ 1.500794000 & -1.558471000 \\ 1.269857000 & -1.541569000 \\ 0.577371000 & -1.618104000 \\ 2.117443000 & -2.433343000 \\ 1.520552000 & 0.818474000\end{array}$

1.520552000

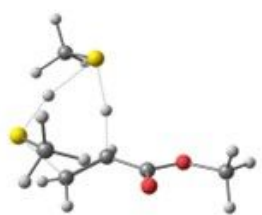

0.305473000

$-0.090920000$

1.303914000

$-0.607393000$

$-0.339204000$

0.604797000

$-1.172765000$

$-0.280060000$

$-0.911559000$

0.965588000

1.982672000

0.840812000

$-0.068464000$

2.200626000

3.258835000

1.973640000

1.950189000

$-1.692773000$

$-0.898044000$

$-2.558589000$

$-3.102913000$

$-1.849619000$

$-3.268316000$
0.285848000

1.091491000

$-0.420491000$

0.356442000

$-0.484572000$

$-0.205995000$

$-0.331134000$

$-1.533806000$

1.789871000

1.506321000

1.312412000

2.439425000

0.226022000

$-0.415605000$

$-1.120176000$

$-0.900768000$

$-1.162928000$

$-2.063469000$

$-0.922408000$

0.084653000

1.069626000

1.096774000

0.044041000
0.459665000

\author{
$E=-745.204940815$ \\ $E-D 3=-745.217365178$ \\ $E_{T H F}=-745.21608697$ \\ $G_{\text {corr }, 298 k, \text { gas }}=0.110873$ \\ $\begin{array}{llll}6 & 1.834896000 & -0.352897000 & 0.578883000\end{array}$
}

\section{Free thiolate anion pathway}

1.222197000

Triethyl amine, $\mathrm{NEt}_{3}$ $E=-292.427409873$ $E-D 3=-292.444984877$ $E_{T H F}=-292.433114231$ $G_{\text {corr }, 298 K, \text { gas }}=0.172708$ 6 $-0.854722000$ $-0.646024000$ $-0.598109000$

$-2.352731000$

$-2.689852000$

$-2.584453000$

$-2.930326000$

1.358087000

1.828108000

1.326748000

2.256203000

2.453788000

1.799155000

3.221964000

$-0.231949000$

$-1.215967000$

0.500722000

$-0.153727000$

$-0.899610000$

0.828209000

$-0.353723000$

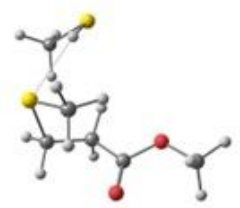

$-0.352897000$

0.578883000

$-0.201613000$

0.456742000

$-1.519542000$

1.173531000

0.691870000

$-0.084134000$

$-1.767150000$

$-1.777091000$

$-2.741180000$

$-0.992118000$

$-0.590551000$

1.264114000

1.888655000

1.385827000

1.943252000

$-0.251272000$

1.716404000

2.128108000

0.661457000

2.256026000

$-1.359603000$

$-0.834928000$

$-0.696073000$

$-0.503680000$

0.217517000

$-1.459465000$

1.196940000

2.297254000

0.971943000

0.637657000

1.022177000

0.814671000

0.175752000

0.420640000

2.254058000

2.640083000

2.933900000

2.284729000

0.712902000

0.051620000

0.291718000

2.112542000

2.550708000
$-0.645836000$

0.137705000

$-1.129647000$

$-1.373389000$

2.198932000

1.229364000

1.546469000

1.954552000

$-0.327845000$

$-1.047782000$

$-1.495643000$

$-1.083550000$

$-1.741895000$

$-2.406244000$

$-0.708469000$

0.635680000

0.890293000

1.581847000

0.740233000

1.322660000
Thiol-amine adduct, $\mathrm{MeSH}$... $\mathrm{NEt}_{3}$
$E=-731.134699856$

$E-D 3=-731.161688017$

$E_{\text {THF }}=-731.145496967$

$\mathrm{G}_{\mathrm{corr}, 298 \mathrm{~K}, \mathrm{gas}}=0.212081$

6

1

1

1

1

6

1

1

6

1

1

1

6
1

1

0.595552000

0.928516000

$-0.454939000$

2.807056000

2.041304000

$-2.724685000$

$-2.929840000$

$-2.491962000$

$-2.483844000$
$-4.001862000$

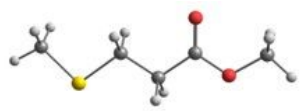

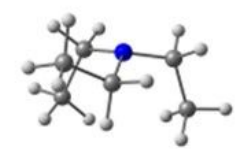

$-0.302343000$

$-0.953358000$

$-0.604468000$

$-2.020154000$

$-0.805868000$

0.230295000

$-1.137545000$

$-1.410912000$

$-0.769636000$

$-0.348566000$

$-1.857032000$

$-0.479210000$

0.590351000

$-0.839014000$

$-0.984908000$

1.135506000

1.286903000

1.483064000

2.028336000

1.736090000

1.987097000

3.070588000

0.582421000

$-0.425256000$

$-1.453824000$

$-0.412406000$

$-0.145981000$

$-0.250540000$

0.870853000

$-0.852314000$

0.619543000

1.517566000

0.773995000

$-0.599301000$

$-0.713130000$

$-1.526871000$

$-0.485769000$

0.752687000

1.215649000

1.490420000

$-0.501041000$

$-1.247321000$

$-0.978939000$

$-0.228224000$

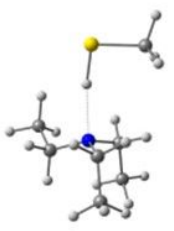

$-0.017916000$

$-0.206255000$

$-0.908819000$

$-0.825533000$

$-1.974074000$

$-0.447330000$

0.581132000

$-0.499605000$

$-1.087181000$

$-0.860236000$

$-0.354354000$

$-1.880445000$

$-0.926687000$

0.064753000

$-1.381671000$

$-1.535478000$

1.257948000

1.634920000

1.861326000

1.540164000

1.587355000

2.954069000

$-0.483768000$

0.943022000

0.733305000

1.850361000

1.107278000
1.623743000 
Methyl thiolate, $\mathrm{CH}_{\mathrm{s}} \mathrm{S}$ $E=-438.110416041$ $E-D 3=-438.111231792$ $E_{T H F}=-438.200108935$ $\mathrm{G}_{\text {corr.,298K,gas }}=0.01231$

$\begin{array}{lr}16 & 0.715396000 \\ 6 & -1.130089000 \\ 1 & -1.556321000 \\ 1 & -1.553608000 \\ 1 & -1.555871000\end{array}$

Triethylammonium, $\mathrm{NEt}_{\mathrm{s}} \mathrm{H}^{+}$ $E=-292.818617416$ $E-D 3=-292.838410069$ $E_{T H F}=-292.904683809$ $\mathrm{G}_{\text {corr.,298K,gas }}=\mathbf{0 . 1 8 9 2 2 8}$

7

$-0.014219000$ $-0.898050000$ $-0.604968000$ $-0.642540000$ $-2.378714000$ $-2.676492000$ $-2.657603000$ $-2.959177000$ 1.425786000 1.894600000 1.345419000 2.229032000 2.439680000 1.745422000 3.189875000 $-0.138055000$ $-1.051260000$ 0.707729000 $-0.182557000$ $-1.091310000$ 0.682251000 $-0.187215000$ $-0.379531000$

MA ‥MeS

$E=-744.598530325$ $E-D 3=-744.609830612$ $E_{\mathrm{THF}}=-\mathbf{7 4 4 . 6 7 4 4 4 1 2 2 5}$ $\mathrm{G}_{\text {corr.,298K,gas }}=0.093418$

$\begin{array}{ll}6 & 1.696884000 \\ 8 & 1.703160000 \\ 8 & 2.848782000 \\ 6 & 3.963497000 \\ 1 & 3.755858000 \\ 1 & 4.787755000 \\ 1 & 4.239894000 \\ 6 & 0.611611000 \\ 1 & 0.828693000 \\ 6 & -0.627289000 \\ 1 & -1.412580000 \\ 1 & -0.818590000 \\ 16 & -2.926887000 \\ 6 & -3.806168000 \\ 1 & -4.745808000 \\ 1 & -4.056227000 \\ 1 & -3.195553000\end{array}$

MA ‥MeS $\cdots$ MeSH $E=-1183.31649358$ $E-D 3=-1183.34086624$ $E_{T H F}=-1183.38684797$ $\mathrm{G}_{\text {corr.,298k,gas }}=0.136117$

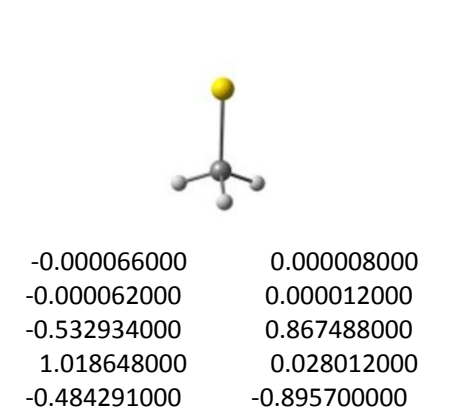

$-0.484291000$

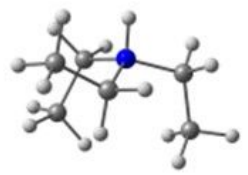

$-0.349647000$

0.607511000

$-0.918017000$

$-0.435654000$

$-1.978125000$

$-0.734633000$

0.316124000

$-1.205862000$

$-1.220903000$

$-0.859382000$

$-0.545682000$

$-1.949709000$

$-0.383955000$

0.685956000

$-0.622697000$

$-0.905474000$

1.169425000

1.322257000

1.454512000

1.991782000

1.812939000

1.830732000

3.046212000

$-0.748419000$

$-0.491469000$

$-1.423449000$

$-0.563153000$

$-0.186958000$

$-0.159211000$

0.761732000

$-0.975028000$

0.539524000

1.475921000

0.552172000

$-0.661029000$

$-0.620740000$

$-1.611220000$

$-0.644468000$

0.796213000

1.425922000

$-0.485462000$

$-1.064047000$

$-1.128992000$

$-0.195581000$

1.477375000

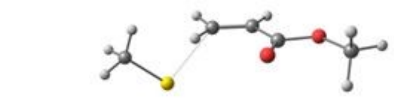

$-0.138443000$

0.153695000

$-1.262952000$

0.641299000

0.020054000

$-0.220296000$

0.736332000

$-0.914201000$

0.554778000

1.549422000

0.007274000

0.488710000

$-0.991965000$

0.537394000

$-0.679188000$

$-0.275590000$

$-1.601877000$

$-0.982459000$

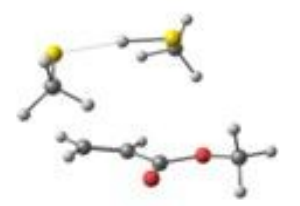

$-0.484227000$

$-1.533472000$

$-0.421288000$

0.019265000

0.816621000

1.194339000

0.924701000

1.488343000

0.555025000

$-0.739812000$

0.319297000

0.724132000

$-0.224100000$

187746000
1.375276000

1.488071000

0.680803000

1.312611000

2.521328000

3.185842000

3.597258000

3.988641000

2.480547000

1.032556000

$-2.804692000$

$-2.448432000$

$-2.706907000$

$-1.383736000$

$-3.025135000$

$-0.522610000$

$-0.859544000$

$-1.174508000$

$-0.201480000$

0.255042000

1.280291000

$-0.421848000$

0.153258000

$-1.365934000$

MA $\cdots M e S \cdots M e S H$ TS

$E=-\mathbf{1 1 8 3 . 3 1 3 8 9 3 7 5}$

$E-D 3=-1183.33668478$

$E_{T H F}=-1183.38142593$

$\mathrm{G}_{\text {corr.,298k,gas }}=0.135041$

6

6

1.988111000

0.951480000

2.484613000

2.456974000

3.461199000

4.349771000

3.724076000

3.100489000

0.680119000

$-1.815502000$

$-1.074392000$

$-0.772864000$

$-0.180345000$

$-1.774588000$

0.300509000

0.705012000

$-0.285957000$

$-1.485475000$

$-3.138175000$

$-3.882605000$

$-3.081552000$

$-3.418488000$

$-0.812517000$

$E=-1183.31644305$

$E-D 3=-1183.34014952$

$E_{\text {THF }}=-1183.38309956$

$\mathrm{G}_{\text {corr, }, 298 \mathrm{k}, \text { gas }}=0.137677$

0.408041000

1.611945000

2.472977000

3.493492000

4.027858000
$-1.116819000$

$-1.317531000$

$-1.621233000$

$-0.248334000$

0.217767000

$-0.614462000$

0.871847000

0.779145000

$-0.842025000$

$-0.066122000$

$-0.889577000$

$-1.956537000$

$-0.812903000$

$-0.438073000$

$-1.911622000$

$-2.344281000$

$-2.013665000$

2.103992000

2.069880000

1.712423000

1.369592000

3.059839000

1.267138000

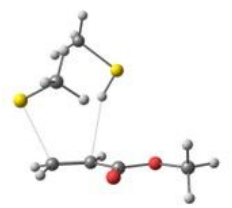

$-0.310714000$

$-0.543469000$

$-1.096089000$

1.002431000

1.317407000

0.685174000

2.365996000

1.185176000

0.285524000

$-1.911723000$

$-1.137961000$

$-1.883116000$

$-0.568922000$

$-0.438491000$

$-1.759429000$

$-2.601172000$

$-1.998904000$

2.196160000

1.465721000

1.921778000

0.385505000

1.642797000

1.027599000

$-0.233856000$

$-1.199627000$

0.572542000

$-0.311808000$

0.638412000

0.518294000

0.469824000

1.665708000

$-1.845015000$

0.046195000

1.529277000

2.274531000

1.244075000

2.002179000

$-1.286918000$

$-0.737649000$

$-2.165140000$

$-0.238085000$

0.114431000

$-0.543615000$

$-0.072038000$

1.156139000

$-0.353223000$

$\mathrm{MeSCH} \mathrm{CH}_{2}-\left(\mathrm{COOCH}_{3}\right) \cdots \mathrm{MeSH}$

4.189822000

3.100030000

0.505011000

$-2.331033000$

$-1.625166000$

$-1.246760000$

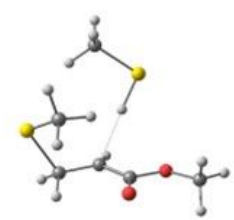

$-0.845449000$

$-0.164412000$

$-813338000$

$-1.547608000$

0.101075000

0.209797000

$-0.738083000$

0.971577000

0.517620000 


$\begin{array}{ll}1 & -0.793285000 \\ 1 & -2.404051000 \\ 6 & -0.650375000 \\ 1 & -0.332213000 \\ 1 & -1.021924000 \\ 16 & -0.105612000 \\ 6 & -1.916181000 \\ 1 & -2.379149000 \\ 1 & -2.347960000 \\ 1 & -2.122995000 \\ 1 & 0.053230000\end{array}$

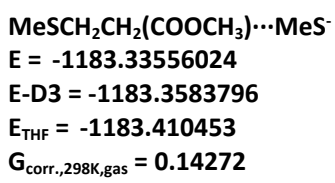

$\begin{array}{lr}6 & 0.054959000 \\ 6 & -0.849181000 \\ 8 & -0.218899000 \\ 8 & 1.212711000 \\ 6 & 2.303591000 \\ 1 & 1.997081000 \\ 1 & 3.089690000 \\ 1 & 2.634507000 \\ 1 & -0.826495000 \\ 16 & -2.691934000 \\ 6 & -1.339265000 \\ 1 & -1.358486000 \\ 1 & -0.361906000 \\ 1 & -1.524820000 \\ 6 & -2.287085000 \\ 1 & -2.512846000 \\ 1 & -2.998127000 \\ 16 & 1.998982000 \\ 6 & 2.005776000 \\ 1 & 1.895761000 \\ 1 & 1.185584000 \\ 1 & 2.941467000 \\ 1 & -0.357457000\end{array}$

\section{Lithium thiolate pathway}

$\mathrm{Et}_{3} \mathrm{NH}^{+} \mathrm{ClO}_{4}$

$E=-1053.76674116$

$E-D 3=-1053.79960986$

$E_{\text {THF }}=-\mathbf{1 0 5 3 . 7 9 5 6 2 7 2 1}$

$\mathrm{G}_{\text {corr.,298K,gas }}=0.195811$

$\begin{array}{lr}7 & 1.208435000 \\ 6 & 1.205180000 \\ 1 & 2.220483000 \\ 1 & 0.551589000 \\ 6 & 0.662485000 \\ 1 & 1.286701000 \\ 1 & -0.359674000 \\ 1 & 0.640312000 \\ 6 & 1.370502000 \\ 1 & 1.159294000 \\ 1 & 0.551669000 \\ 6 & 2.720038000 \\ 1 & 3.529104000 \\ 1 & 2.983143000 \\ 1 & 2.662298000 \\ 6 & 2.059874000 \\ 1 & 1.477708000 \\ 1 & 2.122280000 \\ 6 & 3.449473000 \\ 1 & 3.404852000 \\ 1 & 4.056957000 \\ 1 & 3.965187000 \\ 1 & 0.211477000\end{array}$

$\begin{array}{cc}-0.100478000 & 1.286370000 \\ -0.277410000 & 2.040517000 \\ -1.822451000 & -0.995070000 \\ -2.680552000 & -0.397174000 \\ -2.169966000 & -1.965040000 \\ 2.502354000 & -0.191063000 \\ 2.439711000 & 0.133170000 \\ 3.352939000 & -0.251261000 \\ 1.569630000 & -0.368246000 \\ 2.360472000 & 1.204386000 \\ 1.146258000 & -0.441273000\end{array}$

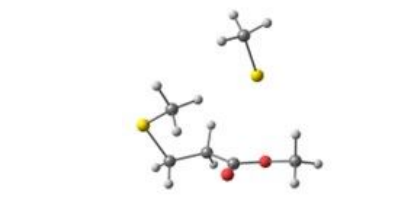

$-1.581391000$

0.079605000

$-1.072949000$

$-1.613262000$

$-2.036489000$

$-2.153652000$

$-2.704255000$

$-2.693159000$

$-1.138203000$

$-1.803563000$

0.922274000

1.323140000

0.641734000

1.267451000

2.348221000

$-0.816897000$

$-1.460916000$

$-1.050820000$

1.228911000

2.912208000

3.700049000

3.052942000

3.118873000

$-0.162067000$
$-1.023246000$

1.264831000

$-0.438266000$

0.490685000

1.385184000

$-0.042221000$

0.743290000

$-1.841175000$

$-0.104357000$

1.066628000

1.918817000

0.565844000

1.399735000

$-0.550960000$

0.304048000

$-1.349866000$

$-0.570675000$

0.180341000

$-0.579703000$

0.901212000

0.721065000

$-1.396561000$

$\begin{array}{ll}8 & -1.548028000 \\ 17 & -2.231385000 \\ 8 & -3.351640000 \\ 8 & -1.168578000 \\ 8 & -2.656028000\end{array}$

$\mathrm{LiClO}_{4}$

$\mathrm{E}=-\mathbf{7 6 8 . 3 1 9 4 1 6 5 6 3}$

$E-D 3=-768.322304436$

$E_{\mathrm{CHCl} 3}=-768.337512448$

$E_{T H F}=-768.344274226$

$G_{\text {corr.,298K,gas }}=-\mathbf{0 . 0 1 1 5 1 6}$

$17-0.195790000$

$8 \quad 0.787082000$

$8-0.998296000$

$8 \quad 0.788503000$

0.788503000
-0.998860000

MA- $\mathrm{LiClO}_{4}$

$E=-1074.83468416$

$E-D 3=-1074.8507108$

$E_{C H C l 3}=-1074.84716209$

$\mathrm{G}_{\text {corr.,298K,gas }}=\mathbf{0 . 0 7 3 5 8 7}$

$17 \quad 0.332752000$

$8 \quad 1.642107000$

$8 \quad 3.803831000$

1.641521000

2.295605000

$-2.404845000$

$-3.823495000$

$-1.496036000$

$-2.203591000$

$-0.833163000$

$-0.306860000$

$-0.911003000$

$-0.306769000$

$-3.973308000$

$-4.842527000$

$-4.673448000$

$-5.870880000$

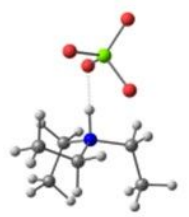

$-0.101052000$

0.703245000

0.709659000

0.158619000

2.113870000

2.710647000

2.089742000

2.620959000

$-1.583328000$

$-2.056085000$

$-1.851094000$

$-2.030205000$

$-1.892606000$

$-1.512738000$

$-3.098513000$

0.469785000

1.289217000

$-0.308689000$

0.958124000

1.806487000

0.176554000

1.295671000

$-0.033655000$
0.121868000

$-1.152815000$

$-1.555339000$

$-1.834967000$

$-0.944729000$

$-0.273519000$

$-0.558582000$

$-1.913289000$

$-0.109440000$

0.853759000

$-0.779685000$

$-0.655829000$

0.065598000

$-1.582387000$

$-0.883102000$

1.229553000

1.658051000

1.994097000

0.831361000

0.144466000

0.372589000

1.735018000

0.515029000
LiSMe

$E=-445.653870033$

$E-D 3=-445.655318533$

$E_{T H F}=-445.680898709$

$\mathrm{G}_{\text {corr.,298K,gas }}=\mathbf{0 . 0 1 1 6 2 7}$

$\begin{array}{lr}16 & -0.440211000 \\ 3 & -1.831498000 \\ 6 & 1.272143000 \\ 1 & 1.852681000 \\ 1 & 1.289423000 \\ 1 & 1.762912000\end{array}$

MA …LiSMe

$E=-752.168659028$

$E-D 3=-752.180489373$

$E_{\text {THF }}=-752.187713694$

$\mathrm{G}_{\text {corr.,298K,gas }}=0.094652$

3
6
6
8
8
6
1
1
1

$-1.096912000$

1.744962000

3.106312000

0.744560000

1.700866000

0.399793000

$-0.168586000$

0.616215000

$-0.168609000$
$-0.769595000$

$-0.107360000$

$-0.923556000$

0.007421000

1.265650000

$-1.054972000$

0.109831000

0.608692000

1.226282000

$-0.263320000$

$\begin{array}{cc}0.000391000 & -0.000605000 \\ 0.000113000 & -0.000125000 \\ -0.012743000 & -1.197233000 \\ 1.227995000 & -0.011593000 \\ 0.011973000 & 1.196814000 \\ -1.227610000 & 0.012504000\end{array}$

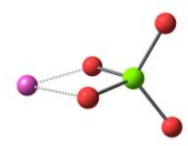

0.012504000

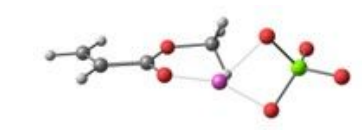

$\begin{array}{cc}-1.373685000 & 0.000059000 \\ -0.017534000 & 0.000051000 \\ -0.595740000 & -1.199489000 \\ -0.483219000 & 0.000620000 \\ -0.594574000 & 1.199718000 \\ 1.455361000 & -0.000698000 \\ -0.316506000 & -0.000120000 \\ -0.723448000 & 0.000036000 \\ -1.154558000 & -0.000277000 \\ 0.991260000 & -0.000113000 \\ 1.472674000 & -0.000121000 \\ 1.130372000 & -0.892909000 \\ 2.557776000 & 0.000039000 \\ 1.129995000 & 0.892452000 \\ -1.798123000 & -0.000073000 \\ 0.141176000 & 0.000299000 \\ 1.213010000 & 0.000396000 \\ -0.205512000 & 0.000403000\end{array}$

$\begin{array}{cc}-0.421011000 & -0.000176000 \\ 1.234622000 & 0.000347000 \\ 0.292252000 & 0.000445000 \\ -0.168581000 & -0.803033000 \\ 1.376386000 & -0.149813000 \\ 0.070987000 & 0.951949000\end{array}$

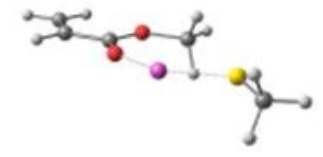

\footnotetext{
$-1.127077000$

$-0.326621000$

$-0.898092000$

$-1.051679000$

0.995639000

1.642483000

1.366461000

2.709072000

1.366451000

0.000033000

$-0.000052000$

$-0.000123000$

$-0.000002000$

$-0.000037000$

$-0.000020000$

0.890811000

$-0.000031000$
} 


$\begin{array}{lr}1 & 3.128026000 \\ 6 & 4.220867000 \\ 1 & 4.180511000 \\ 1 & 5.200668000 \\ 16 & -2.802737000 \\ 6 & -4.444354000 \\ 1 & -5.237419000 \\ 1 & -4.572583000 \\ 1 & -4.572590000\end{array}$

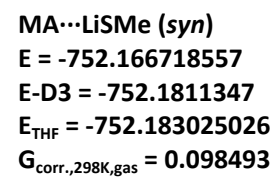

$\begin{array}{lr}3 & -0.699687000 \\ 6 & 1.286431000 \\ 6 & 0.384687000 \\ 8 & 1.052764000 \\ 8 & 2.421510000 \\ 6 & 3.323614000 \\ 1 & 3.654999000 \\ 1 & 4.167349000 \\ 1 & 2.840962000 \\ 1 & 0.558067000 \\ 6 & -0.685177000 \\ 1 & -1.402008000 \\ 1 & -0.743296000 \\ 16 & -2.633715000 \\ 6 & -2.091459000 \\ 1 & -2.789906000 \\ 1 & -2.056878000 \\ 1 & -1.093543000\end{array}$

MA...LiSMe...MeSH $E=-\mathbf{1 1 9 0 . 8 8 8 2 2 4 5 3}$ E-D3 =-1190.90750109 $E_{T H F}=-1190.90569273$ $\mathrm{G}_{\text {corr.,298K,gas }}=0.136066$

$\begin{array}{lr}3 & 0.585291000 \\ 6 & -2.181209000 \\ 6 & -2.094098000 \\ 8 & -1.250018000 \\ 8 & -3.433316000 \\ 6 & -3.635198000 \\ 1 & -3.338064000 \\ 1 & -4.701434000 \\ 1 & -3.049608000 \\ 1 & -3.028627000 \\ 6 & -0.943832000 \\ 1 & 0.002303000 \\ 1 & -0.923195000 \\ 16 & 2.133084000 \\ 6 & 1.431827000 \\ 1 & 2.127879000 \\ 1 & 0.474519000 \\ 1 & 1.267531000 \\ 16 & 2.022046000 \\ 6 & 2.417327000 \\ 1 & 2.463867000 \\ 1 & 3.358349000 \\ 1 & 1.606828000 \\ 1 & 2.999470000\end{array}$

MeS- $\mathrm{CH}_{2} \mathrm{CH}(\mathrm{Li})\left(\mathrm{COOCH}_{3}\right) \cdots \mathrm{MeSH}$

$E=-\mathbf{1 1 9 0 . 8 8 0 5 1 6 9 3}$

$E-D 3=-1190.90260955$

$E_{T H F}=-1190.89451333$

$\mathrm{G}_{\text {corr.,298K,gas }}=0.140189$

\begin{abstract}
$-1.983015000$
$-0.160805000$

0.923522000

$-0.627442000$

0.266522000

$-0.581608000$

0.171384000

$-1.206883000$

$-1.206493000$
\end{abstract}

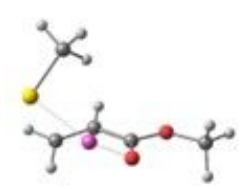

0.692609000

$-0.340597000$

$-1.256192000$

0.244113000

$-0.108572000$

0.853221000

0.529078000

0.906143000

1.830437000

$-1.454766000$

$-1.772160000$

$-2.413794000$

$-1.748500000$

0.275290000

1.046125000

0.782121000

2.137030000

0.683078000

1.511404000

0.064945000

$-0.592293000$

1.149457000

$-0.606681000$

$-0.033103000$

0.956671000

$-0.720669000$

0.055431000

$-1.643442000$

0.074491000

$-0.421546000$

1.155031000

0.396223000

$-1.185547000$

$-1.984183000$

$-1.108846000$

$-1.475394000$

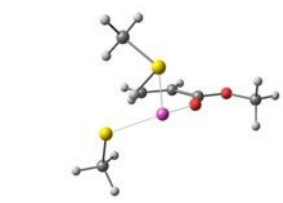

0.227155000

$-0.073880000$

$-1.080518000$

0.358854000

0.356419000

1.345007000

0.946281000

1.566651000

2.242609000

$-1.303286000$

$-1.686562000$

$-1.472137000$

$-2.426397000$

$-1.378942000$

$-3.042695000$

$-3.821474000$

$-3.215278000$

$-3.161983000$

2.284861000

1.741564000

0.651211000

2.186455000

2.105908000

1.585594000
$-0.790016000$

0.180769000

1.252714000

$-0.501132000$

0.006402000

$-1.023540000$

$-1.996641000$

$-1.007254000$

$-0.809802000$

1.758034000

1.573124000

1.069065000

2.367951000

$-0.486018000$

$-0.870136000$

$-0.545582000$

$-1.945224000$

$-0.267857000$

1.451466000

1.492011000

1.777857000

2.085962000

$-0.881739000$
$-0.361146000$

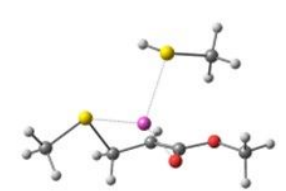

0.520626000

$-1.019374000$

0.282625000

$-0.508119000$

$-1.987422000$

$-2.978329000$

$-2.780107000$

$-3.924046000$

$-3.039223000$

$-0.000818000$

2.332231000

3.836993000

4.625236000

3.612449000

4.187017000

1.705487000

1.918661000

2.362505000

$-0.946675000$

$-2.433626000$

$-2.204696000$

$-2.831539000$

$-3.169163000$

$-0.163386000$
$-1.385390000$

$-1.465551000$

$-1.074849000$

$-1.618615000$

$-1.669787000$

$-2.476824000$

$-1.855933000$

$-0.730595000$

$-1.671526000$

0.413331000

0.134559000

$-0.319012000$

$-0.515249000$

1.101485000

$-1.399917000$

$-1.882859000$

$-1.804953000$

2.539546000

1.728631000

0.720998000

2.344894000

1.673054000

2.501055000
0.020137000

0.993466000

$-1.215933000$

0.448034000

$-0.574270000$

$-1.287325000$

$-0.060063000$

$-1.134797000$

2.017448000

0.327062000

$-0.684690000$

$-0.079100000$

$-1.535626000$

$-1.053284000$

0.619658000

$-0.340548000$

1.390980000

$-0.161660000$

0.567177000

0.915976000

1.374206000

$-0.236822000$

0.934972000

MeS- $\mathrm{CH}_{2} \mathrm{CH}(\mathrm{Li})\left(\mathrm{COOCH}_{3}\right) \cdots \mathrm{MeSH}$ TS

$E=-1190.87462706$

$E-D 3=-1190.89929071$

$E_{T H F}=-\mathbf{1 1 9 0 . 8 8 6 5 9 8 8 8}$

$\mathrm{G}_{\text {corr.,298K,gas }}=0.143601$

$3 \quad 0.304304000$

$-0.477249000$

0.134588000

$-0.090973000$

$-1.678572000$

$-2.412623000$

$-1.829256000$

$-3.299045000$

$-2.716258000$

$-0.349989000$

2.421386000

3.131091000

3.891410000

2.334939000

3.602457000

1.607392000

2.236555000

1.811868000

$-1.039732000$

$-2.764054000$

$-2.752010000$

$-3.381505000$

$-3.176527000$

$-0.550520000$

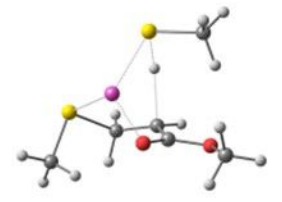

0.551864000

$-1.295441000$

0.302535000

1.350063000

$-0.917743000$

0.617260000

$-0.474476000$

$-1.008687000$

$-0.029582000$

$-1.191412000$

2.319494000

$-0.071460000$

$-0.915302000$

$-0.281177000$

$-1.141401000$

$-1.838944000$

1.403281000

1.547568000

2.243961000

$-0.142606000$

$-0.178421000$

0.080086000

0.540116000

$-1.179963000$

0.729099000

1.60066400

0.540819000

2.142077000

1.243937000

MeS- $\mathrm{CH}_{2} \mathrm{CH}_{2}\left(\mathrm{COOCH}_{3}\right) \cdots$ LiSMe

$E=-1190.90396318$

$E-D 3=-1190.92720477$

$E_{T H F}=-1190.92854892$

$\mathrm{G}_{\text {corr.,298K,gas }}=0.146232$

0.455713000

$-0.088157000$

$-0.163839000$

0.000868000

$-0.098561000$

0.067299000

$-0.751519000$

0.057584000

1.016213000

$-0.107503000$

$-1.752518000$

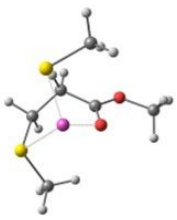

1.010894000

1.196919000

$-1.220700000$

$-0.228311000$

$-0.269874000$

$-1.386700000$

$-0.858864000$

0.947920000

$-2.505658000$

$-3.459761000$

$-0.575301000$

$-3.371754000$

$-4.437612000$

$-3.287122000$

$-0.825396000$

1.667706000

0.602742000

0.496988000

1.215132000

0.017678000

1.009903000

$-2.326080000$

0.079461000

0.971132000 


$\begin{array}{lccc}1 & -3.834302000 & 0.427104000 & 0.355551000 \\ 1 & -2.490860000 & -0.344843000 & 1.260847000 \\ 1 & -3.247604000 & 1.146569000 & 1.870293000 \\ 6 & -1.422261000 & 0.624089000 & -1.411468000 \\ 1 & -2.326995000 & 0.038230000 & -1.599267000 \\ 1 & -1.309971000 & 1.330772000 & -2.236747000 \\ 16 & 2.311902000 & 1.747996000 & 0.167677000 \\ 6 & 3.270448000 & 0.167628000 & 0.070108000 \\ 1 & 2.692822000 & -0.647760000 & -0.390529000 \\ 1 & 4.163090000 & 0.316781000 & -0.544109000 \\ 1 & 3.595035000 & -0.169933000 & 1.059602000 \\ 1 & 0.718527000 & 0.392490000 & -1.288683000\end{array}$

\section{Sodium thiolate pathway}

$\mathrm{NaClO}_{4}$

$E=-923.079848958$

$E-D 3=-923.083718113$

$E_{\mathrm{CHCl3}}=-923.099637109$

$E_{\mathrm{THF}}=-923.105940358$

$G_{\text {corr.,298k, gas }}=-\mathbf{0 . 0 1 4 3 8 6}$

$\begin{array}{lc}11 & 2.251038000 \\ 17 & -0.548492000 \\ 8 & 0.396577000 \\ 8 & -1.360105000 \\ 8 & 0.395822000 \\ 8 & -1.361925000\end{array}$

$\mathrm{MA}-\mathrm{NaClO}_{4}$

$E=-1229.5871974$

$E-D 3=-1229.60525088$

$E_{\mathrm{CHCl} 3}=-\mathbf{1 2 2 9 . 6 0 2 3 3 4 8 5}$

$\mathrm{G}_{\text {corr.,298k, gas }}=0.070297$

$6 \quad-2.5639990000$

$6 \quad-4.013034000$

$8-1.720992000$

$8-2.268465000$

$6-0.864766000$

$1-0.364305000$

$1-0.855770000$

$1-0.364382000$

$1-4.245965000$

$6 \quad-4.964399000$

$1-4.714752000$

$1-6.016110000$

$11 \quad 0.373288000$

$17 \quad 2.453564000$

$8 \quad 1.801485000$

$8 \quad 3.905114000$

$8 \quad 1.801667000$

$8 \quad 2.128906000$

\section{NaSMe}

$E=-600.420161541$

$E-D 3=-600.422239199$

$E_{\text {THF }}=-600.443792133$

$\mathrm{G}_{\mathrm{corr}, 298 \mathrm{~K}, \text { gas }}=0.009515$

$16 \quad 0.363630000$

$11-1.909479000$

$6 \quad 1.595884000$

12.598958000

$1 \quad 1.506064000$

$1 \quad 1.505857000$

MA -..NaSMe

$E=-906.922460759$

$E-D 3=-906.935801368$

$E_{\text {THF }}=-906.941784297$

$\mathrm{G}_{\text {corr.,298k,gas }}=0.091004$

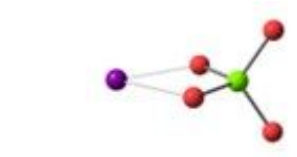

0.000034000

0.000082000

0.015011000

1.228629000

$-0.016468000$

$-1.227395000$

$-0.000025000$

$-0.000127000$

$-1.212677000$

0.015350000

1.213377000

$-0.015746000$

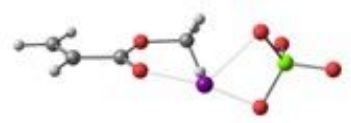

$$
\begin{array}{r}
-0.217122000 \\
-0.512122000 \\
-1.117182000 \\
1.074976000 \\
1.448813000 \\
1.067644000 \\
2.536402000 \\
1.068087000 \\
-1.572218000 \\
0.425907000 \\
1.481863000 \\
0.157747000 \\
-1.769492000 \\
0.189448000 \\
-0.477233000 \\
-0.059565000 \\
-0.477380000 \\
1.635311000
\end{array}
$$

$-0.000084000$

$-0.000238000$

0.000161000

$-0.000139000$

$-0.000158000$

$-0.891521000$

$-0.000446000$

0.891444000

$-0.000695000$

0.000315000

0.000772000

0.000267000

$-0.000035000$

0.000039000

$-1.212962000$

$-0.000101000$

1.213023000

0.000130000

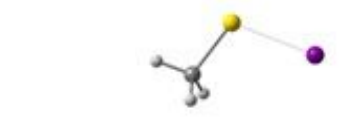

$-0.685589000$

0.351167000

0.697930000

0.261667000

1.328513000

1.328830000

0.000001000

0.000001000

$-0.000006000$

0.000234000

$-0.889893000$

0.889661000

$\begin{array}{lr}11 & -1.128512000 \\ 6 & 1.978544000 \\ 6 & 3.386346000 \\ 8 & 1.039965000 \\ 8 & 1.833748000 \\ 6 & 0.483812000 \\ 1 & -0.062199000 \\ 1 & 0.608077000 \\ 1 & -0.062242000 \\ 1 & 3.497814000 \\ 6 & 4.437840000 \\ 1 & 4.308817000 \\ 1 & 5.452593000 \\ 16 & -2.752617000 \\ 6 & -4.539389000 \\ 1 & -5.149054000 \\ 1 & -4.805455000 \\ 1 & -4.805451000\end{array}$

MA ‥NaSMe (syn)

$E=-906.921835704$

$E-D 3=-906.938032154$

$E_{\text {THF }}=-\mathbf{9 0 6 . 9 3 9 0 2 6 9 2 4}$

$G_{\text {corr.,298k,gas }}=0.095727$

$11 \quad 0.762314000$

$6 \quad-1.428449000$

$6-0.515834000$

$-1.249339000$

$-2.535873000$

$-3.470064000$

$-3.839147000$

$-4.286868000$

$-3.006042000$

$-0.698755000$

2.686643000

2.046213000

2.620151000

2.128602000

0.991130000

0.553358000

0.641765000

1.247782000

MA...NaSMe...MeSH

$E=-1345.63528953$

$E-D 3=-1345.66180216$

$E_{\text {THF }}=-1345.65208495$

$\mathrm{G}_{\text {corr.,298k,gas }}=0.133874$

$11-0.604796000$

$6 \quad 1.568380000$

0.753003000

1.185749000

2.822402000

3.673956000

3.780086000

4.635947000

3.263447000

1.197881000

$-2.579741000$

$-3.782790000$

$-4.481386000$

$-3.282548000$

$-4.364249000$

$-0.522996000$

$-0.937013000$

$-1.165654000$

0.215990000

0.503671000

$-0.222320000$

0.430361000

1.513955000
$-1.504210000$

$-0.229800000$

$-0.685932000$

$-1.027200000$

1.089295000

1.624595000

1.303371000

2.705813000

1.303276000

$-1.765649000$

0.138072000

1.215415000

$-0.247103000$

0.436216000

$-0.030202000$

0.878557000

$-0.611953000$

$-0.612028000$

0.000001000

0.000065000

$-0.000016000$

0.000047000

0.000020000

0.000025000

0.889229000

$-0.000035000$

$-0.889119000$

$-0.000004000$

$-0.000091000$

$-0.000090000$

$-0.000123000$

$-0.000007000$

$-0.000020000$

0.000020000

$-0.888378000$

0.888290000

$\begin{array}{cc}1.820742000 & -0.282184000 \\ -0.270162000 & -0.294444000 \\ -1.352689000 & -0.596539000 \\ 0.935163000 & -0.556276000 \\ -0.679124000 & 0.355794000 \\ 0.344659000 & 0.725394000 \\ 0.872129000 & -0.158464000 \\ -0.171800000 & 1.229668000 \\ 1.070028000 & 1.400500000 \\ -2.300393000 & -0.103278000 \\ 0.104818000 & 0.020709000 \\ -0.645029000 & 1.575238000 \\ -1.544641000 & 1.815860000 \\ 0.044552000 & 2.421232000 \\ -0.939801000 & 1.465589000 \\ -1.165885000 & -1.415074000 \\ -0.281797000 & -2.032828000 \\ -1.967216000 & -1.629208000\end{array}$

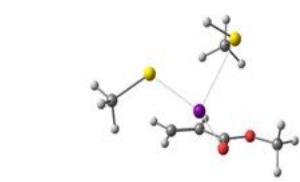

0.302097000

$-1.221930000$

$-1.753412000$

$-0.995523000$

$-0.926972000$

$-0.332474000$

$-0.993686000$

$-0.183880000$

0.624980000

$-1.750322000$

0.152143000

$-1.196324000$

$-1.303964000$

$-2.163207000$

$-0.981022000$

$-2.128420000$

$-2.175312000$

$-2.453377000$

2.696247000

1.712538000

0.899264000

2.357950000

1.304705000
1.565394000

$-0.002292000$

$-1.088311000$

1.152624000

$-0.397657000$

0.596651000

1.460420000

0.106856000

0.930712000

$-2.077333000$

$-0.124181000$

0.233953000

$-0.601100000$

0.377475000

1.135333000

$-0.872885000$

0.124924000

$-1.682177000$

0.022215000

$-1.512187000$

$-1.571622000$

$-2.388570000$

$-1.445347000$ 


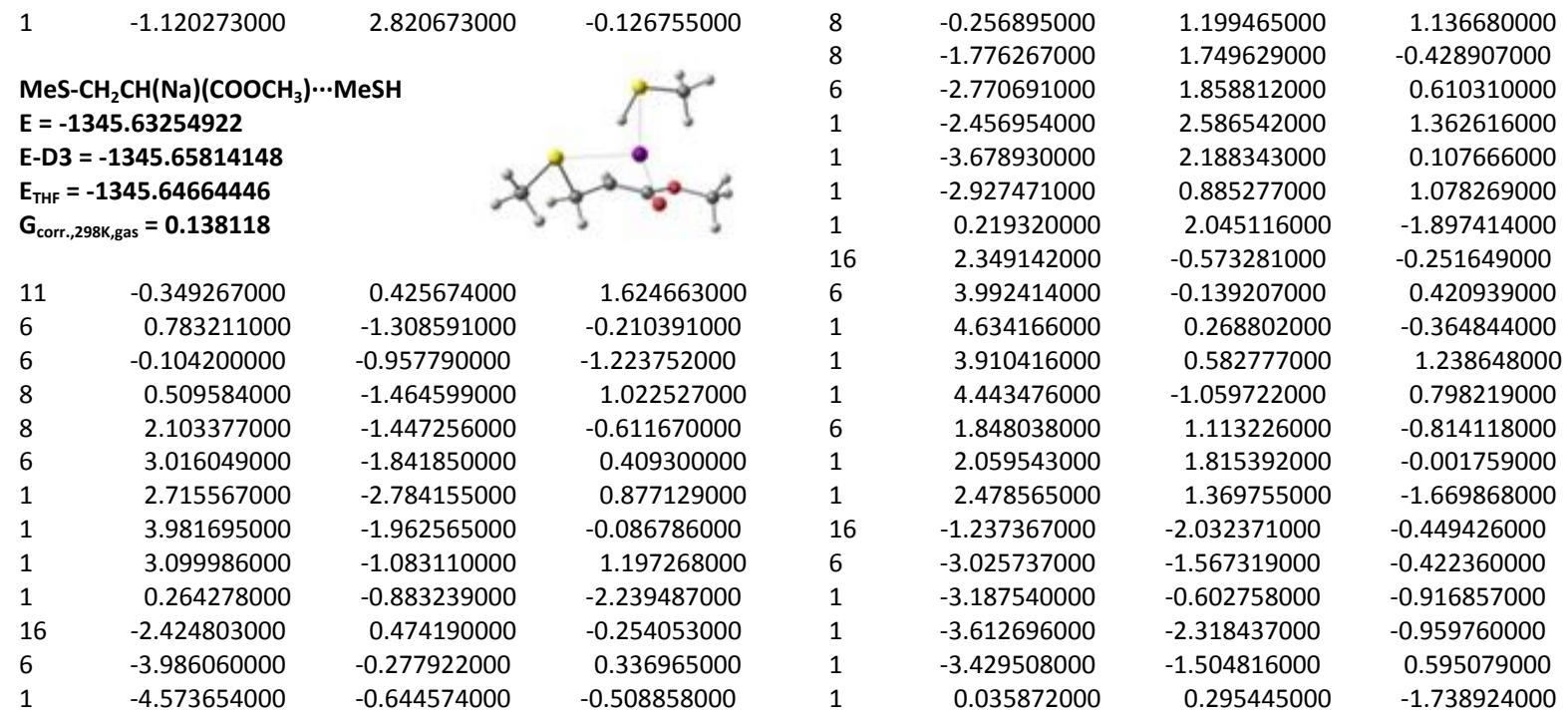

$\begin{array}{lll}-4.562737000 & 0.489628000 & 0.858029000\end{array}$

$-1.556985000 \quad-1.092006000 \quad-0.986775000$

$-1.785163000-1.862573000-0.242225000$

$-2.109687000 \quad-1.295581000-1.906314000$

$2.305969000 \quad-0.153267000$

$1.904811000-0.292434000$

$0.826255000 \quad-0.389224000$

$2.403072000 \quad-1.165790000$

2.673350000

0.606616000

3.097446000

2.261879000

0.430371000

1.211991000

MeS- $\mathrm{CH}_{2} \mathrm{CH}(\mathrm{Na})\left(\mathrm{COOCH}_{3}\right) \cdots$ MeSH TS

$\mathrm{E}=\mathbf{- 1 3 4 5 . 6 3 0 7 4 5}$

$E-D 3=-1345.6565456$

$E_{T H F}=-1345.64543954$

$\mathrm{G}_{\text {corr.,298K,gas }}=0.138251$

$\begin{array}{lrrr}11 & -0.334707000 & 0.475783000 & 1.699879000 \\ 6 & 0.894498000 & -1.225105000 & -0.220476000 \\ 6 & 0.042173000 & -0.693753000 & -1.219930000 \\ 8 & 0.586800000 & -1.396487000 & 0.989590000 \\ 8 & 2.179586000 & -1.461603000 & -0.628511000 \\ 6 & 3.110191000 & -1.795489000 & 0.403277000 \\ 1 & 2.782986000 & -2.672404000 & 0.968175000 \\ 1 & 4.053247000 & -2.003164000 & -0.105057000 \\ 1 & 3.242701000 & -0.960446000 & 1.101328000 \\ 1 & 0.431385000 & -0.696862000 & -2.233311000 \\ 16 & -2.439568000 & 0.383430000 & -0.176071000 \\ 6 & -3.892222000 & -0.631270000 & 0.273031000 \\ 1 & -4.399574000 & -0.987206000 & -0.627744000 \\ 1 & -3.599315000 & -1.488973000 & 0.886001000 \\ 1 & -4.582778000 & -0.001717000 & 0.838471000 \\ 6 & -1.427112000 & -0.961922000 & -1.067390000 \\ 1 & -1.614935000 & -1.857727000 & -0.465326000 \\ 1 & -1.908433000 & -1.079477000 & -2.040673000 \\ 16 & 0.637217000 & 2.257514000 & -0.177791000 \\ 6 & 2.466822000 & 2.022209000 & -0.265465000 \\ 1 & 2.690158000 & 0.981572000 & -0.508497000 \\ 1 & 2.884454000 & 2.664906000 & -1.043154000 \\ 1 & 2.927936000 & 2.280768000 & 0.690338000 \\ 1 & 0.314374000 & 0.908730000 & -0.804332000 \\ & & & \end{array}$

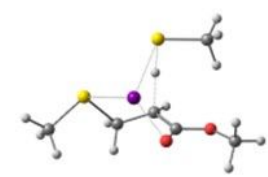

4. Potassium thiolate pathway

$\mathrm{KClO}_{4}$

$E=-1360.69085888$

$E-D 3=-1360.69571468$

$E_{\mathrm{CHCl} 3}=-1360.71134833$

$E_{T H F}=-1360.71672307$

$G_{\text {corr.,298k,gas }}=-\mathbf{0 . 0 1 6 1 9 2}$

$19-2.280126000$

$17 \quad 0.919070000$

$8-0.007396000$

$8 \quad 1.738730000$

$8-0.007466000$

$\begin{array}{r}-0.00746600000 \\ \hline\end{array}$

$\mathrm{MA}-\mathrm{KClO}_{4}$

$E=-1667.19037292$

$E-D 3=-1667.21035656$

$E_{\mathrm{CHCl} 3}=-1667.20718873$

$\mathrm{G}_{\text {corr.,298k,gas }}=\mathbf{0 . 0 6 8 7 2 6}$

$6 \quad-2.679073000$

$6-4.144150000$

$-1.883300000$

$-2.319951000$

$-0.898134000$

$-0.417808000$

$-0.825800000$

$-0.417930000$

$-4.432806000$

$-5.047293000$

$-4.744322000$

$-6.111298000$

0.447071000

2.443960000

1.853831000

3.913632000

1.854618000

2.021195000

$-0.075168000$

$-0.291942000$

$-1.013560000$

1.203091000

1.498267000

1.089600000

2.583156000

1.090600000

$-1.338610000$

0.692222000

1.734112000

0.477329000

$-2.119787000$

0.472667000

$-0.229042000$

0.333025000

$-0.229978000$

1.894482000
0.000886000 0.000155000 0.001332000 0.000177000 0.000098000 $-0.889913000$ $-0.000519000$ 0.890631000 0.000728000 $-0.001133000$ $-0.001709000$ $-0.001575000$ $-0.000450000$ $-0.000028000$ $-1.215268000$ $-0.000549000$

1.215051000 0.000674000
MeS- $\mathrm{CH}_{2} \mathrm{CH}_{2}\left(\mathrm{COOCH}_{3}\right) \cdots \mathrm{NaSMe}$

$\mathrm{E}=\mathbf{- 1 3 4 5 . 6 5 5 0 5 5 0 4}$

$E-D 3=-1345.68106385$

$E_{T H F}=-1345.67952341$

$\mathrm{G}_{\text {corr.,298K,gas }}=0.142154$

\section{KSMe}

$E=-1038.02604581$

$E-D 3=-1038.02842841$

$E_{T H F}=-1038.05164168$

$\mathrm{G}_{\text {corr.,298K,gas }}=0.007768$

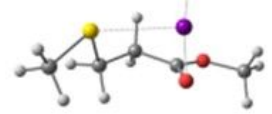

$11 \quad 0.077187000$

$6 \quad-0.561669000$

6

0.374241000
-1.040740000
1.366326000

1.200513000
1.519252000

$-0.041822000$

$-1.217587000$
0.933438000 $-1.818533000$

2.093141000

3.122584000
$-0.684910000$ 0.171669000 0.756689000 0.385920000
0.000000000 0.000001000 $-0.000009000$ 0.000914000 


$\begin{array}{ll}1 & 1.968403000 \\ 1 & 1.967282000 \\ \text { MA } \cdots \text { KSMe } \\ \text { E = -1344.52277909 } \\ \text { E-D3 = -1344.53696374 } \\ \mathbf{E}_{\text {THF }}=-\mathbf{1 3 4 4 . 5 4 3 4 5 9 9} \\ \mathbf{G}_{\text {corr.,298K,gas }}=\mathbf{0 . 0 8 8 9 3 7} \\ \\ 19 & -1.176204000 \\ 6 & 2.175178000 \\ 6 & 3.607897000 \\ 8 & 1.280143000 \\ 8 & 1.963594000 \\ 6 & 0.584047000 \\ 1 & 0.056223000 \\ 1 & 0.641863000 \\ 1 & 0.055928000 \\ 1 & 3.777333000 \\ 6 & 4.614875000 \\ 1 & 4.428467000 \\ 1 & 5.648813000 \\ 16 & -2.652861000 \\ 6 & -4.497534000 \\ 1 & -4.883927000 \\ 1 & -4.893777000 \\ 1 & -4.893959000\end{array}$

$\begin{array}{lrlrrr}1.384884000 & -0.889154000 & 1 & 1.110127000 & -0.603909000 & -2.380948000 \\ 1.385900000 & 0.888279000 & 16 & -2.739839000 & 0.197050000 & -0.185374000 \\ & & 6 & -3.857908000 & -1.160982000 & -0.719351000 \\ & & 1 & -4.346061000 & -0.902179000 & -1.663684000 \\ & & 1 & -3.314382000 & -2.104262000 & -0.868797000 \\ & & 1 & -4.638500000 & -1.339214000 & 0.026542000 \\ & & 6 & -0.591345000 & -1.463213000 & -1.420859000 \\ -1.808308000 & -0.000035000 & 1 & -0.962458000 & -2.014599000 & -0.567010000 \\ -0.092309000 & -0.000208000 & 6 & -1.265133000 & -1.359592000 & -2.260755000 \\ -0.471210000 & 0.000015000 & 1 & 1.059444000 & 2.575329000 & 0.053778000 \\ -0.935336000 & -0.000152000 & 1 & 1.992053000 & 1.453387000 & -0.992725000 \\ 1.219554000 & -0.000379000 & 1 & 1.366166000 & 2.793371000 & -1.995682000 \\ 1.675001000 & -0.000278000 & 1 & -0.761263000 & 1.793758000 & -0.696386000\end{array}$

MA...KSMe (syn)

$E=-1344.52322401$

$E-D 3=-1344.54110206$

$E_{T H F}=-1344.54177849$

$\mathrm{G}_{\text {corr.,298K,gas }}=0.093745$

$19 \quad 0.935020000$

$6-1.542161000$

$6 \quad-0.689725000$

$8-1.313715000$

$8-2.677285000$

$6 \quad-3.562530000$

$1 \quad-3.883445000$

$1-4.418906000$

$1 \quad-3.080695000$

$1-0.946182000$

$16 \quad 2.597241000$

$6 \quad 1.793240000$

2.211319000

1.935490000

0.710988000

0.414786000

0.565239000

1.031294000

1.324667000
2.761637000

1.324331000

$-1.543629000$

0.406297000

1.475233000

0.075653000

0.712171000

0.683589000

1.707628000

0.177943000

0.888284000

$-0.000504000$

$-0.888533000$

$-0.000136000$

0.000500000

0.000792000

0.000818000

0.000234000

0.000043000

0.000147000

$-0.887695000$

0.177696000

0.887560000

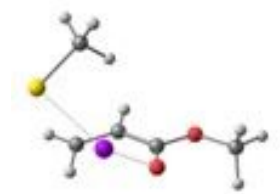

$-0.070931000$

$-0.368492000$

$-0.325531000$

$-1.468779000$

0.841338000

$-0.708229000$

$-0.775861000$

0.261921000

0.880081000

$-0.244739000$

0.908124000

$-2.447308000$

$-0.492059000$

$-1.153361000$

$-2.131091000$

$-0.493281000$

501405000

0.296164000

0.725967000

$-0.117823000$

1.172308000

1.467423000

$-0.318720000$

0.030974000

1.545111000

1.802280000

$-1.285137000$

2.408446000

1.382471000

$-1.277322000$

$-1.485510000$

$-0.344496000$

$-2.013383000$

$-2.107985000$

$-1.799818000$
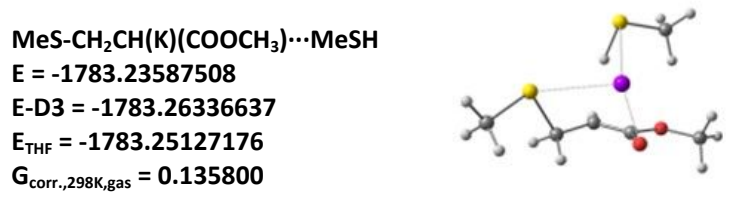

$19-0.335852000$

0.819682000

$-0.056309000$

0.543948000

2.150005000

3.070257000

2.771410000

4.031803000

3.170009000

0.328191000

$-2.442224000$

$-4.079490000$

$-4.532364000$

$-3.993999000$

$-4.727861000$

$-1.511535000$

$-1.732705000$

$-2.034092000$

0.947416000

2.724485000

2.837903000

3.066841000

3.311790000

0.447027000

0.406043000

$-1.372423000$

$-0.940679000$

$-1.649287000$

$-1.451784000$

$-1.869983000$

$-2.818445000$

$-1.983756000$

$-1.125129000$

$-0.804804000$

0.443300000

$-0.308159000$

$-0.651904000$

$-1.155854000$

0.450575000

$-1.109280000$

$-1.877555000$

$-1.345650000$

2.310962000

1.892294000

0.808145000

2.307639000

2.324692000

1.146962000
2.017153000

$-0.321416000$

$-1.317549000$

0.882084000

$-0.728922000$

0.271148000

0.727354000

$-0.235081000$

1.073043000

$-2.321443000$

$-0.457634000$

$-0.132180000$

$-1.066407000$

0.555498000

0.312207000

$-1.119722000$

$-0.370778000$

$-2.050254000$

$-0.385135000$

$-0.653404000$

$-0.666252000$

$-1.602914000$

0.159053000

$-0.942187000$

MeS- $\mathrm{CH}_{2} \mathrm{CH}(\mathrm{K})\left(\mathrm{COOCH}_{3}\right) \cdots \mathrm{MeSH}$ TS

$E=-1783.23427625$

$E-D 3=-1783.26187051$

$E_{\text {THF }}=-1783.25054941$

$\mathrm{G}_{\text {corr, } 298 \mathrm{k}, \text { gas }}=0.135581$

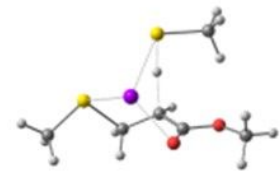

0.621799000

2.025299000

$-0.331551000$

$-1.306221000$

$-0.251499000$

0.085746000

$-0.825679000$

$-1.261571000$

0.675059000

$-1.479973000$

0.957771000

3.811805000

3.831303000

4.780313000

3.555049000

$-1.192878000$

$-1.504018000$

0.880034000

$-0.932859000$

$-1.928737000$

$-0.692541000$

$-0.201922000$
1.489803000

$-0.660367000$

0.421399000

0.872014000

$-0.017366000$

1.194623000
2.255052000

$-1.494676000$

3.206429000

$-1.777767000$

2.907679000

$-2.643435000$

4.147563000

$-1.979800000$

$-0.919493000$

$-0.869323000$

0.468716000

0.284707000

$-0.683494000$

$-3.974337000$

$-4.362189000$

$-3.785458000$

$-4.725513000$

$-1.372636000$

$-1.527238000$

$-1.834378000$
$-1.100593000$

$-1.499362000$

$-0.011250000$

$-1.132898000$

$-1.939553000$

$-1.415332000$

2.221058000

1.997001000
$-0.675226000$

0.348621000

0.946346000

$-0.166136000$

1.020673000

$-2.276833000$

$-0.433012000$

$-0.117298000$

$-1.051022000$

0.587937000

0.303573000

$-1.087874000$

$-0.362526000$

$-2.037496000$

$-0.559445000$

2.475552000

$-0.723966000$ 


\begin{tabular}{|c|c|c|c|}
\hline 1 & 2.707404000 & 0.933091000 & -0.808782000 \\
\hline 1 & 2.835639000 & 2.508696000 & -1.619127000 \\
\hline 1 & 2.988819000 & 2.412908000 & 0.146638000 \\
\hline 1 & 0.323637000 & 0.788578000 & -0.983462000 \\
\hline \multicolumn{4}{|c|}{$\mathrm{MeS}-\mathrm{CH}_{2} \mathrm{CH}_{2}\left(\mathrm{COOCH}_{3}\right) \cdots \mathrm{KSMe}$} \\
\hline \multicolumn{4}{|c|}{$E-D 3=-1783.28435209$} \\
\hline \multicolumn{4}{|c|}{$E_{T H F}=-1783.28037515$} \\
\hline \multicolumn{4}{|c|}{$G_{\text {corr.,298K,gas }}=0.141715$} \\
\hline 19 & 0.077064000 & -1.576570000 & 1.549821000 \\
\hline 6 & -0.520303000 & 1.438922000 & 0.254044000 \\
\hline 6 & 0.391294000 & 1.632977000 & -0.937093000 \\
\hline 8 & -0.166890000 & 1.044660000 & 1.360917000 \\
\hline 8 & -1.766972000 & 1.828833000 & -0.022977000 \\
\hline 6 & -2.755130000 & 1.529139000 & 0.977531000 \\
\hline 1 & -2.480713000 & 1.972107000 & 1.938433000 \\
\hline 1 & -3.686798000 & 1.954979000 & 0.606866000 \\
\hline 1 & -2.845943000 & 0.443459000 & 1.072979000 \\
\hline 1 & 0.241287000 & 2.653404000 & -1.309027000 \\
\hline 16 & 2.351622000 & -0.369274000 & -0.610513000 \\
\hline 6 & 4.071749000 & -0.153977000 & -0.029544000 \\
\hline 1 & 4.646094000 & 0.452412000 & -0.735747000 \\
\hline 1 & 4.104958000 & 0.312486000 & 0.960038000 \\
\hline 1 & 4.525796000 & -1.146136000 & 0.026718000 \\
\hline 6 & 1.869231000 & 1.412377000 & -0.616875000 \\
\hline 1 & 2.107968000 & 1.832541000 & 0.365213000 \\
\hline 1 & 2.487120000 & 1.908809000 & -1.370670000 \\
\hline 16 & -1.376403000 & -1.541050000 & -0.981082000 \\
\hline 6 & -3.107108000 & -1.031405000 & -1.350438000 \\
\hline 1 & -3.169705000 & 0.055084000 & -1.482217000 \\
\hline 1 & -3.449376000 & -1.501269000 & -2.277416000 \\
\hline 1 & -3.804584000 & -1.320486000 & -0.555040000 \\
\hline 1 & 0.028667000 & 0.946484000 & -1.710478000 \\
\hline
\end{tabular}

\section{Other optimized enolate structures and related systems}

MeS- $\mathrm{CH}_{2} \mathrm{CH}=\mathrm{C}(\mathrm{OH}) \mathrm{OCH}_{3}$ $E=-745.162347939$

$E-D 3=-745.174896984$

$E_{T H F}=-745.171150123$

$\mathrm{G}_{\text {corr.,298K,gas }}=0.111389$

$\begin{array}{lr}6 & -1.274948000 \\ 8 & -0.968130000 \\ 8 & -2.595050000 \\ 6 & -3.411688000 \\ 1 & -3.052881000 \\ 1 & -4.409756000 \\ 1 & -3.441422000 \\ 6 & -0.377687000 \\ 1 & -0.728950000 \\ 6 & 1.064050000 \\ 1 & 1.614422000 \\ 1 & 1.198864000 \\ 16 & 1.876404000 \\ 6 & 3.379801000 \\ 1 & 3.913871000 \\ 1 & 3.122325000 \\ 1 & 4.030935000 \\ 1 & -0.041611000\end{array}$

$\mathrm{Li}\left(\mathrm{OMe}_{2}\right)_{3}(\mathrm{SMe})$

$E=-910.828132793$

$E-D 3=-910.861571766$

$E_{\text {THF }}=-910.846016672$

$\mathrm{G}_{\text {corr, } 298 \mathrm{k}, \text { gas }}=\mathbf{0 . 2 3 3 7 3 8}$

$\begin{array}{lr}16 & -1.044730000 \\ 6 & -2.662112000 \\ 1 & -3.039452000 \\ 1 & -2.579810000\end{array}$

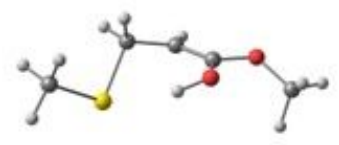

$-0.276723000$

0.966934000

$-0.486248000$

0.655829000

1.189428000

0.257874000

1.349452000

$-1.259422000$

$-2.179023000$

$-1.108510000$

$-2.034587000$

$-0.830251000$

0.260060000

0.534622000

1.383634000

0.767502000

$-0.343186000$

1.157929000

0.186192000

0.628836000

0.010353000

$-0.275747000$

$-1.163287000$

$-0.464941000$

0.568728000

$-0.034680000$

$-0.483175000$

0.333376000

0.154450000

1.386916000

$-0.654479000$

0.350177000

$-0.082413000$

1.387461000

0.323333000

0.375177000

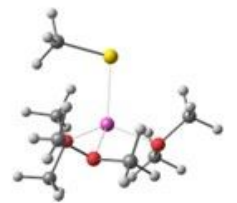

0.848357000

$-1.800650000$

$-0.036371000$

$-0.306637000$

$-0.961929000$
$-1.653431000$

$-2.644748000$

$-1.067644000$

$\begin{array}{cc}1 & -3.420213000 \\ 3 & 0.108550000 \\ 8 & 2.032871000 \\ 8 & -0.488120000 \\ 8 & -0.002082000 \\ 6 & 2.209422000 \\ 1 & 1.496915000 \\ 1 & 3.235012000 \\ 1 & 1.986381000 \\ 6 & -1.914222000 \\ 1 & -2.142356000 \\ 1 & -2.306913000 \\ 1 & -2.366318000 \\ 6 & -0.142779000 \\ 1 & -0.334256000 \\ 1 & 0.776583000 \\ 1 & -0.983811000 \\ 6 & 2.896847000 \\ 1 & 2.697636000 \\ 1 & 2.702687000 \\ 1 & 3.949656000 \\ 6 & 0.232379000 \\ 1 & 0.291011000 \\ 1 & -0.588053000 \\ 1 & 1.173937000 \\ 6 & -0.064198000 \\ 1 & 1.026171000 \\ 1 & -0.471498000 \\ 1 & -0.386653000\end{array}$

$\mathrm{Li}\left(\mathrm{OMe}_{2}\right)_{3}(\mathrm{SMe})-\mathrm{MA}$ adduct $E=-1217.30267871$

E-D3 $=\mathbf{- 1 2 1 7 . 3 5 9 9 2 4 1 9}$

$E_{T H F}=-1217.31685184$

$\mathrm{G}_{\text {corr. }, 298 \mathrm{~K}, \text { gas }}=0.324397$

$3 \quad 0.921429000$

$6-2.630081000$

$6-2.620381000$

$-1.678859000$

$-3.841316000$

$-3.934419000$

$-3.665245000$

$-4.974105000$

$-3.274159000$

$-3.493112000$

0.432989000

$-0.946247000$

$-1.199194000$

$-0.697682000$

$-1.844674000$

$-1.517821000$

$-0.698279000$

$-1.471217000$

2.998083000

1.181158000

0.694702000

0.302580000

0.928286000

$-0.749359000$

0.473967000

3.586091000

4.679069000

3.171582000

3.340574000

0.372375000

$-0.287529000$

$-0.245270000$

1.002429000

$-0.052627000$

$-1.114985000$

0.342672000
0.593212000

0.131926000

0.636791000

0.796259000

$-1.783831000$

1.650012000

2.445005000

2.041426000

1.253546000

0.671371000

$-0.387674000$

1.012753000

1.241956000

$-2.644576000$

$-1.990001000$

$-3.225494000$

$-3.335954000$

$-0.476964000$

$-1.192535000$

$-0.954785000$

$-0.166277000$

$-2.459220000$

$-1.692646000$

$-3.153056000$

$-3.027176000$

2.155289000

2.152187000

2.764642000

2.568956000
$-1.173753000$

0.032966000

$-0.124220000$

1.800780000

0.348848000

$-1.123484000$

$-0.901105000$

$-1.088661000$

$-2.121140000$

1.832441000

1.697033000

2.799872000

1.012708000

$-0.781038000$

$-1.635120000$

$-0.942451000$

$-0.637741000$

$-0.326920000$

0.472765000

$-1.297518000$

$-0.283513000$

1.574763000

2.350243000

1.805494000

1.542637000

1.932487000

1.880244000

1.114170000

2.897553000

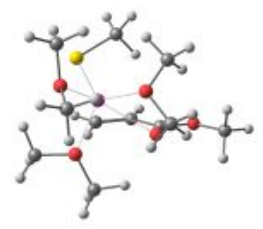

$-0.084416000$

$-0.095512000$

$-1.543779000$

0.660480000

0.381738000

1.800516000

2.307576000

2.007638000

2.163699000

$-2.072683000$

$-2.126569000$

$-1.890824000$

$-2.839681000$

$-1.172019000$

$-1.527384000$

$-2.186499000$

$-1.636042000$

$-3.267001000$

0.039420000

$-0.153117000$

1.737650000

2.865403000

2.867570000

2.786563000

3.796430000

1.199600000

1.098874000

1.304349000

2.093454000

0.409884000

$-0.350336000$

1.178199000

0.838411000

1.612526000

1.450251000

0.740710000
0.061490000

$-0.419148000$

$-0.526288000$

$-0.629881000$

$-0.022416000$

0.117587000

$-0.814288000$

0.374374000

0.912348000

$-0.160082000$

1.165420000

2.347354000

2.829918000

3.135834000

1.827916000

$-0.976121000$

$-1.411702000$

$-1.025841000$

0.294115000

$-1.936921000$

0.884439000

0.107356000

$-0.788414000$

$-0.184981000$

0.666211000

$-0.274560000$

$-0.337666000$

$-1.279243000$

0.316006000

$-2.966141000$

$-3.405197000$

$-2.504288000$

$-3.758442000$

2.093916000

1.876642000

2.616449000 


0.069001000
3.464091000
2.912556000
4.545081000
3.261663000
1.967711000
2.549738000
1.324961000
2.643335000

\begin{abstract}
2.509724000
$-0.219307000$

$-1.091008000$

$-0.418492000$

0.640122000

$-1.258536000$

$-1.596025000$

$-2.074140000$

$-0.950616000$
\end{abstract}

2.717020000

1.618467000

1.975179000

1.613977000

2.274673000

$-2.381930000$

$-1.524595000$

$-2.738527000$

$-3.192104000$
MeS- $\mathrm{CH}_{2} \mathrm{CH}=\mathrm{C}(\mathrm{OMe})\left[\mathrm{OLi}\left(\mathrm{Me}_{2} \mathrm{O}\right)_{3}\right]$ $E=-1217.30615607$ $E-D 3=-1217.3556789$ $E_{T H F}=-1217.32421649$ $\mathrm{G}_{\text {corr.,298K,gas }}=0.322711$

\begin{tabular}{|c|c|}
\hline 3 & -1.380374000 \\
\hline 8 & -1.333047000 \\
\hline 8 & -1.615236000 \\
\hline 8 & -3.090217000 \\
\hline 6 & 0.018350000 \\
\hline 1 & 0.534712000 \\
\hline 1 & 0.030560000 \\
\hline 1 & 0.523674000 \\
\hline 6 & -1.460615000 \\
\hline 1 & -2.320880000 \\
\hline 1 & -1.454159000 \\
\hline 1 & -0.539036000 \\
\hline 6 & -3.276172000 \\
\hline 1 & -2.282036000 \\
\hline 1 & -3.896425000 \\
\hline 1 & -3.761786000 \\
\hline 6 & -2.127708000 \\
\hline 1 & -3.135730000 \\
\hline 1 & -1.733036000 \\
\hline 1 & -2.163013000 \\
\hline 6 & -4.301083000 \\
\hline 1 & -4.023623000 \\
\hline 1 & -4.864257000 \\
\hline 1 & -4.936078000 \\
\hline 6 & -0.548478000 \\
\hline 1 & -0.754308000 \\
\hline 1 & 0.410213000 \\
\hline 1 & -0.520684000 \\
\hline 6 & 0.775760000 \\
\hline 8 & 0.242345000 \\
\hline 8 & -0.012202000 \\
\hline 6 & -0.348734000 \\
\hline 1 & 0.557007000 \\
\hline 1 & -0.936315000 \\
\hline 1 & -0.940384000 \\
\hline 6 & 1.976331000 \\
\hline 1 & 2.288743000 \\
\hline 6 & 2.926180000 \\
\hline 1 & 3.440587000 \\
\hline 1 & 2.406405000 \\
\hline 16 & 4.267784000 \\
\hline 6 & 5.322834000 \\
\hline 1 & 6.184048000 \\
\hline 1 & 4.784185000 \\
\hline 1 & 5.685069000 \\
\hline
\end{tabular}

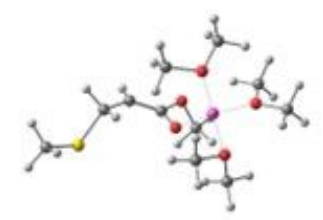

$-0.479568000$

$-0.916570000$

$-2.049915000$

0.438618000

$-1.018191000$

$-1.711580000$

$-1.408714000$

$-0.046151000$

$-1.524588000$

$-0.877395000$

$-2.339382000$

$-0.935189000$

1.818477000

2.235182000

1.930851000

2.342075000

$-0.075898000$

$-0.068629000$

0.949690000

$-0.464210000$

$-0.272540000$

$-1.300062000$

0.166103000

$-0.263741000$

$-2.942156000$

3.305453000

$-2.409506000$

$-3.794651000$

1.208051000

0.115668000

2.379774000

2.640620000

2.782578000

3.563314000

1.826668000

1.392430000

2.402425000

0.267626000

0.319390000

$-0.691660000$

0.270918000

$-1.075178000$

$-1.178932000$

2.028483000

$-0.843030000$
$-0.014533000$

$-1.965100000$

1.192839000

0.222004000

$-2.436954000$

$-1.773862000$

$-3.463214000$

$-2.387819000$

2.518228000

2.703497000

3.254157000

2.585584000

$-0.092930000$

$-0.264963000$

$-0.993383000$

0.741251000

$-2.795519000$

$-2.377936000$

$-2.813457000$

$-3.822440000$

0.446252000

0.688928000

1.281405000

$-0.451530000$

0.848450000

$-0.161228000$

0.866218000

1.540013000

0.078193000

0.500200000

0.225666000

1.583667000

2.189759000

1.598348000

2.022497000

$-0.537048000$

$-0.773091000$

$-0.747031000$

$-1.715342000$

$-0.671153000$

0.554542000

$-0.095651000$

0.569420000

$-0.120661000$
$-1.102890000$

$\mathrm{Na}\left(\mathrm{OMe}_{2}\right)_{3}(\mathrm{SMe})$

$E=-1065.57964362$

$E-D 3=-1065.60773973$

$E_{\text {THF }}=-\mathbf{1 0 6 5 . 5 9 7 2 2 6 0 5}$

$\mathrm{G}_{\text {corr.,298K,gas }}=0.227017$

16

0.822909000

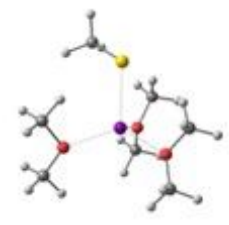

$0.928717000 \quad-2.101580000$

$\begin{array}{lll}6 & 2.563260000 & 1.291441000\end{array}$

$1 \quad 3.108310000$

1.761175000

$-1.591632000$

$-2.416384000$

$\mathrm{Na}\left(\mathrm{OMe}_{2}\right)_{3}(\mathrm{SMe})-\mathrm{MA}$ adduct $E=-1372.06419563$ $E-D 3=-1372.11881599$ $E_{T H F}=-1372.07759684$ $\mathrm{G}_{\text {corr.,298K,gas }}=0.317632$

$11 \quad 0.622172000$

$6-2.525903000$

$-2.609330000$

$-1.496549000$

$-3.727414000$

$-3.719801000$

$-3.302706000$

$-4.762632000$

$-3.134144000$

$-3.542311000$

0.099482000

$-1.177934000$

$-1.568526000$

$-0.783241000$

$-2.017938000$

$-1.519504000$

$-0.686285000$

$-1.616914000$

2.982647000

1.228421000

0.659675000

0.263684000

1.030918000

$-0.701970000$

0.207146000

3.586329000

4.683639000

3.271676000

3.252071000

0.285629000

$-0.182477000$

$-0.484522000$

0.769417000

$-0.234434000$

$-1.238761000$
1.975674000

0.378156000

$-0.147746000$

$-2.111779000$

1.454824000

$-0.292324000$

$-2.372379000$

$-1.626214000$

$-2.275165000$

$-3.385481000$

2.732932000

2.606517000

3.479318000

3.068246000

0.483786000

1.161251000

$-0.163604000$

1.057451000

$-2.988327000$

$-2.707771000$

$-4.034932000$

$-2.905097000$

$-1.255069000$

$-1.798790000$

$-0.776613000$

$-1.968357000$

1.465463000

0.454115000

1.749771000

2.163235000
$-0.734693000$

$-1.316734000$

0.077746000

0.443511000

1.726678000

$-0.389024000$

$-0.645677000$

$-1.417451000$

$-0.317255000$

$-1.040192000$

1.168611000

0.080850000

1.452728000

1.519646000

$-1.542393000$

$-1.734222000$

$-2.417915000$

$-1.360958000$

1.539934000

2.317124000

1.251899000

1.945978000

$-0.063173000$

0.819584000

0.168483000

$-0.886700000$

3.142295000

3.472426000

3.574700000

3.512044000

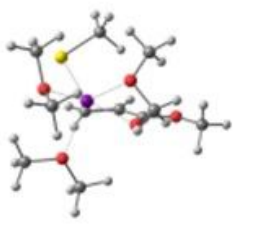

$-0.057849000$

0.031212000

.258784000

422878000

$-1.552861000$

0.183464000

$-0.861445000$

$0.383044000-0.479074000$

$1.680898000-1.071153000$

$1.654061000-2.082966000$

$2.000293000-1.102514000$

$2.388842000 \quad-0.474270000$

$-1.810625000 \quad 0.671072000$

$-1.702429000 \quad 2.155053000$

$-0.712496000 \quad 3.010408000$

$-1.244860000 \quad 3.882733000$

$0.251617000 \quad 3.348039000$

$-0.519127000 \quad 2.323625000$

$-2.392185000 \quad 0.232899000$

$-2.250492000 \quad-0.443921000$

$-3.384164000 \quad 0.653670000$

$0.166655000 \quad 0.314424000$

$-1.120527000$

2.355276000

2.981019000

2.746161000

2.589785000

4.072762000

1.123508000

1.077263000

0.879002000

2.139927000

$-1.331784000$

$-2.322930000$

$-0.567600000$

$-1.252022000$

2.643405000

2.250639000

$-1.976754000$

0.269220000

$-0.948945000$

$-1.691351000$

$-1.290659000$

$-0.828941000$

$-0.543460000$

$-0.483672000$

$-1.561550000$

$-0.292619000$

$-3.023215000$ 


$\begin{array}{lrcc}1 & 0.162411000 & 2.152602000 & 2.231910000 \\ 1 & -0.295968000 & 3.726312000 & 1.521679000 \\ 6 & 3.326028000 & 0.354087000 & 1.686037000 \\ 1 & 2.752969000 & -0.380949000 & 2.255913000 \\ 1 & 4.404001000 & 0.203778000 & 1.842135000 \\ 1 & 3.054563000 & 1.366480000 & 2.020328000 \\ 6 & 2.251713000 & -2.110058000 & -1.938896000 \\ 1 & 2.912116000 & -1.847808000 & -1.110804000 \\ 1 & 1.826379000 & -3.109544000 & -1.768736000 \\ 1 & 2.822371000 & -2.122975000 & -2.879073000\end{array}$

\begin{tabular}{|c|c|c|c|c|c|}
\hline & & & & & 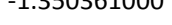 \\
\hline & $\mathrm{CH}=\mathrm{C}(\mathrm{OMe})[\mathrm{ON}$ & $\left.\left.\mathrm{e}_{2} \mathrm{O}\right)_{3}\right]$ & & 1 & -2.897727000 \\
\hline & .06159219 & & & 1 & -1.553283000 \\
\hline & 372.11055201 & & & 6 & -2.724813000 \\
\hline & 72.07417545 & & & 1 & -1.982319000 \\
\hline & gas $=0.31915$ & & & 1 & -2.898648000 \\
\hline & & & & 1 & -3.673135000 \\
\hline 8 & 2.749612000 & -1.844130000 & -0.734770000 & 6 & 3.762096000 \\
\hline 8 & 0.184934000 & 2.148723000 & 0.414511000 & 1 & 3.161680000 \\
\hline 8 & 3.359334000 & 1.394861000 & -0.217724000 & 1 & 4.398464000 \\
\hline 6 & 2.144931000 & -2.256942000 & -1.966226000 & 1 & 4.414740000 \\
\hline 1 & 2.363678000 & -1.480021000 & -2.703990000 & 6 & -3.083669000 \\
\hline 1 & 2.580722000 & -3.205647000 & -2.310736000 & 1 & -2.612266000 \\
\hline 1 & 1.058504000 & -2.345452000 & -1.848330000 & 1 & -4.050884000 \\
\hline 6 & -0.600535000 & 2.078086000 & 1.605995000 & 1 & -3.272851000 \\
\hline 1 & 0.080997000 & 1.846638000 & 2.428821000 & 6 & -1.767436000 \\
\hline 1 & -1.089428000 & 3.042706000 & 1.804158000 & 1 & -1.312437000 \\
\hline 1 & -1.364488000 & 1.294549000 & 1.528462000 & 1 & -1.504171000 \\
\hline 6 & 4.630304000 & 0.871692000 & -0.590360000 & 1 & -2.862460000 \\
\hline
\end{tabular}

$\begin{array}{rcc}2.345738000 & -2.320170000 & 0.868248000 \\ 0.174675000 & 0.694514000 & -0.340476000 \\ 2.873401000 & 1.067081000 & -0.545308000 \\ -1.274393000 & -1.192383000 & -1.684724000 \\ -2.205126000 & 1.631819000 & 0.650050000 \\ 3.558806000 & 0.459171000 & 0.556097000 \\ 2.795314000 & 0.066695000 & 1.238299000 \\ 4.200453000 & -0.362062000 & 0.205791000 \\ 4.182232000 & 1.199736000 & 1.078182000 \\ -1.803408000 & -2.159569000 & -0.773359000 \\ -1.350361000 & -1.965956000 & 0.206152000 \\ -2.897727000 & -2.067505000 & -0.703124000 \\ -1.553283000 & -3.178608000 & -1.101134000 \\ -2.724813000 & 0.787943000 & 1.681187000 \\ -1.982319000 & 0.007212000 & 1.872954000 \\ -2.898648000 & 1.362431000 & 2.602343000 \\ -3.673135000 & 0.328544000 & 1.364188000 \\ 3.762096000 & 1.608877000 & -1.504245000 \\ 3.161680000 & 2.048475000 & -2.306519000 \\ 4.398464000 & 2.393622000 & -1.067304000 \\ 4.414740000 & 0.833422000 & -1.933779000 \\ -3.083669000 & 2.690853000 & 0.321507000 \\ -2.612266000 & 3.282868000 & -0.468596000 \\ -4.050884000 & 2.314549000 & -0.046498000 \\ -3.272851000 & 3.342814000 & 1.187971000 \\ -1.767436000 & -1.357510000 & -3.000225000 \\ -1.312437000 & -0.584430000 & -3.626595000 \\ -1.504171000 & -2.344947000 & -3.408991000 \\ -2.862460000 & -1.247227000 & -3.038997000\end{array}$

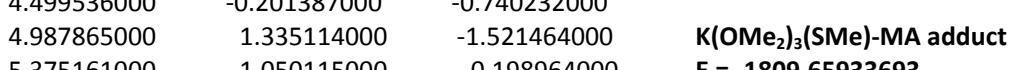

$\begin{array}{lll}5.375161000 & 1.050115000 & 0.198964000\end{array}$

$\begin{array}{lll}2.616648000 & -2.840171000 & 0.277941000\end{array}$

$3.129944000 \quad-2.465778000 \quad 1.167803000$

$\begin{array}{lll}1.561893000 & -3.014930000 & 0.521261000\end{array}$

$\begin{array}{lll}1.090292000 & -3.781140000 & -0.037345000\end{array}$

$\begin{array}{lll}3.376254000 & 2.802699000 & 0.002315000\end{array}$

$\begin{array}{lll}2.359038000 & 3.091470000 & 0.272215000\end{array}$

$\begin{array}{lll}4.068002000 & 3.063784000 & 0.816448000\end{array}$

$\begin{array}{lll}3.686085000 & 3.337546000 & -0.907280000\end{array}$

$-0.631853000$

0.045432000

2.396703000

2.496826000

$-0.737790000$

$-1.591046000$

$1.566705000 \quad-0.910331000$

$3.333352000-0.616199000$

$-1.193458000$

1.435710000

0.122468000

$-0.051928000$

0.379081000

$-0.444409000$

0.076896000

$-1.094216000$

$\begin{array}{rr}-0.841695000 & -0.758156000 \\ -1.138858000 & 1.392339000\end{array}$

$-1.404719000 \quad 2.738930000$

$-0.277604000$

0.652405000

$-2.378006000$

2.836484000

$-1.416511000 \quad 3.315000000$

$-0.942930000$

3.144496000

$-0.631341000$

0.731946000

-2.258330000
-2.557740000

$-1.285118000$

$-1.463805000$

1.755248000

$-3.320925000$

$-1.148564000$

$-0.297319000$

$-4.139509000$

$-0.160742000$

$-1.266663000 \quad-1.300826000$

-2.903834000
-4.105050000

0.555620000

$-0.225276000$

$-5.372709000$

$-5.928687000$

$-4.910716000$

0.380133000

$-1.533879000$

$1.319154000 \quad-1.596329000$

$0.178937000 \quad-2.505709000$

$-6.073529000$

$E-D 3=-1809.7140908$

$E_{\text {THF }}=-1809.67416304$

$\mathrm{G}_{\text {corr.,298K,gas }}=0.312953$

$19-0.705447000 \quad 0.78$

$6 \quad 2.389269000$

2.389269000
1.893577000

1.722297000

3.755972000

4.344692000

3.935114000

5.413795000

4.182495000

2.624730000

$-0.692217000$

0.867996000

1.074255000

0.840860000

1.706622000

0.555566000

$-0.137321000$

0.225991000

$-3.485980000$

$-1.382394000$

1.117808000

1.779384000

1.034787000

2.189372000

2.585573000

$-0.426975000 \quad-1.296584000$

$\mathrm{K}\left(\mathrm{OMe}_{2}\right)_{3}(\mathrm{SMe})$

$E=-1503.17319776$

$E-D 3=-1503.20337835$

$E_{\text {THF }}=-1503.19123308$

$\mathrm{G}_{\text {corr.,298k,gas }}=0.22439$

$\begin{array}{ll}16 & 0.497608000 \\ 6 & 1.352696000 \\ 1 & 1.492597000 \\ 1 & 0.782083000\end{array}$

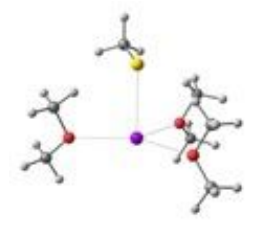

$-1.159391000$

2.039990000

1.247203000

$-2.595710000$

1.973297000

$-3.402668000$

0.404182000

$-4.462752000$

$-5.180841000$

$-3.941540000$

$-5.021534000$

$-0.422554000$

$-0.166446000$

0.476522000

$-0.800842000$

2.019457000

2.470005000

1.444738000

2.817059000

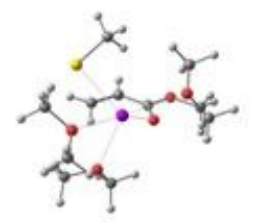

0.782588000

0.196179000

109757000

0.081146000

$-2.076656000$

$-0.353293000$

$-0.856274000$

$-1.079568000 \quad 0.118207000$

$-0.182881000 \quad 1.057503000$

$-0.332395000 \quad 2.061652000$

$-0.401688000 \quad 1.050146000$

$0.861729000 \quad 0.770052000$

$-2.544089000 \quad-1.506175000$

$-0.701160000 \quad-2.576492000$

$0.032309000 \quad-3.188730000$

$-0.282357000-4.216081000$

$1.127807000-3.169138000$

$-0.303361000-2.556115000$

$-2.367746000 \quad-0.967434000$

$-2.058880000 \quad-0.196377000$

$-3.171178000-1.611958000$

$0.900097000 \quad 0.021410000$

$-1.326258000$

1.837913000

$2.948319000 \quad 0.426502000$

$\begin{array}{ll}2.845828000 & 1.684395000\end{array}$

$3.052680000 \quad 2.458715000$

$1.838574000 \quad 1.830411000$

$3.590139000 \quad 1.769069000$

$\begin{array}{ll}1.239547000 & 0.990010000\end{array}$

$0.419155000 \quad 1.141725000$

$1.429354000 \quad 1.932270000$

$2.142149000 \quad 0.700402000$

$-2.064391000 \quad 2.586859000$

$-3.004809000 \quad 2.078539000$

$-1.449764000 \quad 2.645699000$

$-2.293852000 \quad 3.594224000$

$2.784371000-0.664459000$

$1.783262000 \quad-0.662996000$

$2.913497000 \quad-1.584551000$

$3.541692000-0.634774000$ 


$\begin{array}{llcl}6 & -4.052783000 & 0.616424000 & -1.259381000 \\ 1 & -3.226840000 & 0.369062000 & -1.931176000 \\ 1 & -4.744198000 & -0.236877000 & -1.198579000 \\ 1 & -4.600034000 & 1.488524000 & -1.646126000 \\ 6 & -2.564220000 & -2.066141000 & 1.567521000 \\ 1 & -3.212652000 & -1.419348000 & 0.973429000 \\ 1 & -2.338874000 & -2.976674000 & 0.992948000 \\ 1 & -3.079269000 & -2.352793000 & 2.496978000\end{array}$

\section{$\mathrm{MeS}-\mathrm{CH}_{2} \mathrm{CH}=\mathrm{C}(\mathrm{OMe})\left[\mathrm{OK}\left(\mathrm{Me}_{2} \mathrm{O}\right)_{3}\right]$}

$E=-1809.6478451$

$E-D 3=-1809.70259296$

$E_{T H F}=-1809.66409391$

$\mathrm{G}_{\text {corr.,298K,gas }}=0.316664$

$19 \quad 0.025274000$

2.517315000

$-2.477818000$

$-0.940875000$

3.568319000

3.430260000

3.538674000

4.549473000

$-2.564571000$

$-1.544085000$

$-3.051442000$

$-3.127009000$

$-0.619813000$

0.078734000

$-0.172812000$

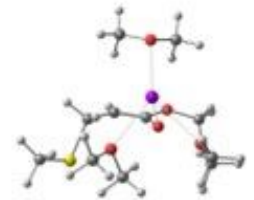

$-1.175322000$

1.278899000

$-0.408018000$

2.201099000

0.180188000

0.787573000

$-3.141816000$

$-0.371189000$

$-0.798349000$

1.316012000

$-1.860979000$

1.095882000

$-0.226498000$

0.380490000

1.795656000

$-0.146280000-0.644059000$

$-0.074246000 \quad-1.026013000$

$-1.059895000-1.017615000$

$0.733950000 \quad-0.975917000$

$-2.956435000$

$-2.118694000$

$-1.753152000$

$-1.807669000$

$-2.172233000$

$-1.523493000$

$-2.708670000$

$-2.328816000$

$\begin{array}{cc}0.985374000 & 2.511891000 \\ 1.209781000 & 3.202062000 \\ 1.223325000 & 3.010692000 \\ 1.589264000 & 1.599546000 \\ -4.118819000 & -0.171879000 \\ -4.195476000 & 0.903928000 \\ -3.836449000 & -0.671670000 \\ -5.104514000 & -0.549588000 \\ -0.204044000 & 1.406526000 \\ -0.229126000 & 2.488906000 \\ 0.692513000 & 1.153901000 \\ -1.092494000 & 1.107256000 \\ 0.766317000 & -1.109820000 \\ -0.234333000 & -0.936951000 \\ 0.532759000 & -1.775948000 \\ -0.716762000 & -2.436207000 \\ -0.839322000 & -3.204088000 \\ -0.728919000 & -2.912702000 \\ -1.562383000 & -1.740851000 \\ 2.058416000 & -0.662760000 \\ 2.803728000 & -0.957496000 \\ 2.479652000 & 0.073038000 \\ 3.256410000 & 0.819384000 \\ 1.638086000 & 0.581024000 \\ 3.205041000 & -1.031713000 \\ 3.474025000 & 0.250079000 \\ 3.863554000 & -0.232108000 \\ 2.529220000 & 0.749287000 \\ 4.197263000 & 1.002400000\end{array}$

0.985374000

2.594215000

3.545466000

2.483833000

$-1.945173000$

$-2.128224000$

$-2.884633000$

$-1.633596000$

$-3.751022000$

$-3.595277000$

$-4.335732000$

$-4.329226000$

1.525741000

0.750000000

2.738808000

2.840206000

2.065031000

3.824281000

2.761387000

1.370036000

2.098969000

0.158749000

0.367018000

$-0.326721000$

$-1.191794000$

$-2.469769000$

$-3.370151000$

$-2.712052000$

$-2.136198000$
2.511891000

1.599546000

-

$-0.671670000$

0.549588000

1.153901000

107256000

09820000

$-1.775948000$

.436207000

$-1.740851000$

62760000

0.073038000

0.819384000

024000

0.250079000

1.002400000

Table S3. Composition, glass transition temperature and ionic conductivity of SPEs.

\begin{tabular}{|c|c|c|c|c|c|c|c|}
\hline PE & $\begin{array}{l}\text { Thiol } \\
\text { group: } \\
\text { vinyl } \\
\text { group }\end{array}$ & $\begin{array}{c}\text { Thiol } \\
\text { compound : } \\
\text { PEGDA }\end{array}$ & $\begin{array}{c}\mathbf{E O}: \\
\mathbf{L i}^{+}\end{array}$ & $\begin{array}{c}\text { Decomposition } \\
\text { temperature } \\
\left({ }^{\circ} \mathrm{C}\right)\end{array}$ & $\begin{array}{c}T_{\mathrm{g}} \\
\left({ }^{\circ} \mathbf{C}\right)\end{array}$ & $\begin{array}{c}\sigma\left(\mathrm{S} \mathrm{cm}^{-1}\right) \\
\text { at } 30^{\circ} \mathrm{C}\end{array}$ & $\begin{array}{c}\sigma\left(\mathrm{S} \mathrm{cm}^{-1}\right) \\
\text { at } 60^{\circ} \mathrm{C}\end{array}$ \\
\hline PEGDA $_{200}-\mathrm{S}-\mathrm{POSS}$ & $1: 1$ & $1: 4$ & $16: 1$ & 318 & -15.5 & $2.1 \times 10^{-6}$ & $7.5 \times 10^{-6}$ \\
\hline PEGDA $_{400}-\mathrm{S}-\mathrm{POSS}$ & $1: 1$ & $1: 4$ & $16: 1$ & 307 & -25.5 & $4.9 \times 10^{-6}$ & $8.4 \times 10^{-5}$ \\
\hline PEGDA $_{600}-$ S-POSS & $1: 1$ & $1: 4$ & $16: 1$ & 307 & -32.7 & $5.0 \times 10^{-5}$ & $2.3 \times 10^{-4}$ \\
\hline PEGDA $_{600}$-TMPTMP & $1: 1$ & $2: 3$ & $16: 1$ & 306 & -33.5 & $1.8 \times 10^{-5}$ & $1.7 \times 10^{-4}$ \\
\hline PEGDA $_{600}$-PETMP & $1: 1$ & $1: 2$ & $16: 1$ & 290 & -33.4 & $1.9 \times 10^{-5}$ & $1.7 \times 10^{-4}$ \\
\hline
\end{tabular}




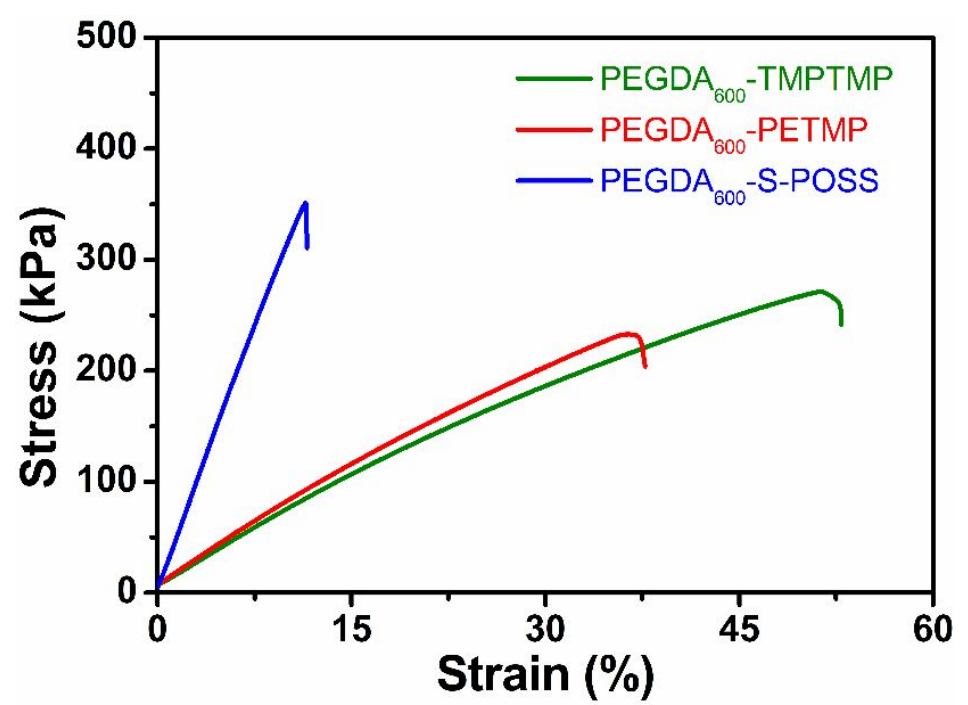

Figure S16. Stress-strain profile of PEGDA $600^{-T M P T M P, ~ P E G D A ~} 600^{-}$PETMP and PEGDA $_{600^{-}}$ S-POSS.

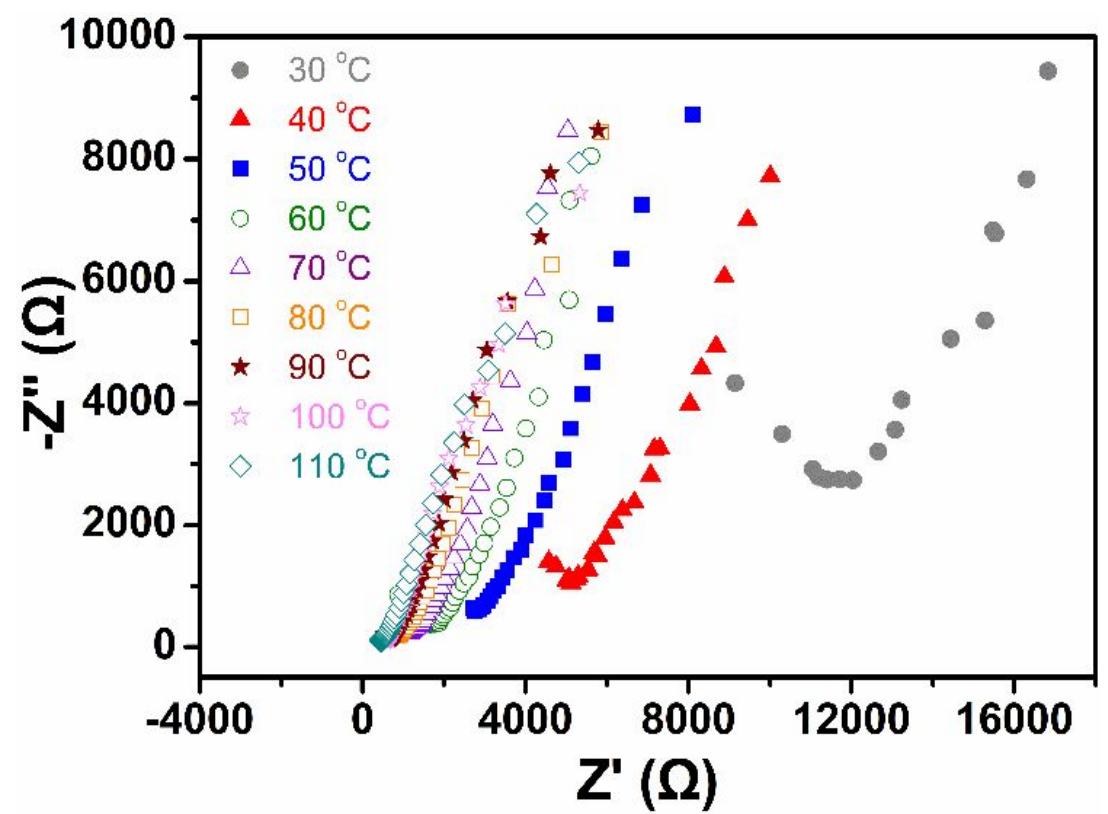

Figure S17. EIS plots of PEGDA $600^{-S-P O S S}$ at $30{ }^{\circ} \mathrm{C}, 40{ }^{\circ} \mathrm{C}, 50^{\circ} \mathrm{C}, 60^{\circ} \mathrm{C}, 70{ }^{\circ} \mathrm{C}, 80^{\circ} \mathrm{C}, 90$ ${ }^{\circ} \mathrm{C}, 100^{\circ} \mathrm{C}$ and $110^{\circ} \mathrm{C}$. 


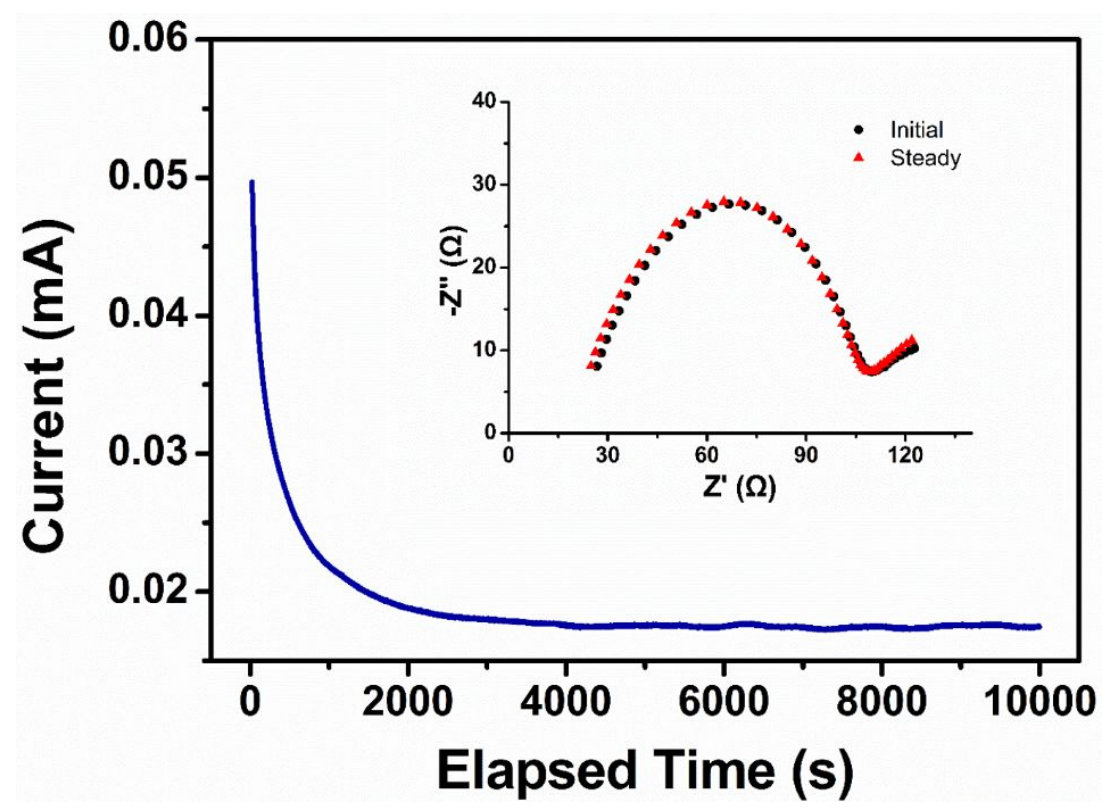

Figure S18. Chronoamperometry of the Li $\mid$ PEGDA $_{600}$-S-POSS $\mid \mathrm{Li}$ cell under the potential step of $10 \mathrm{mV}$. The inset shows the Nyquist plot of the AC impedance of PEGDA $600-\mathrm{S}-\mathrm{POSS}$ before and after polarization of $60^{\circ} \mathrm{C}$.

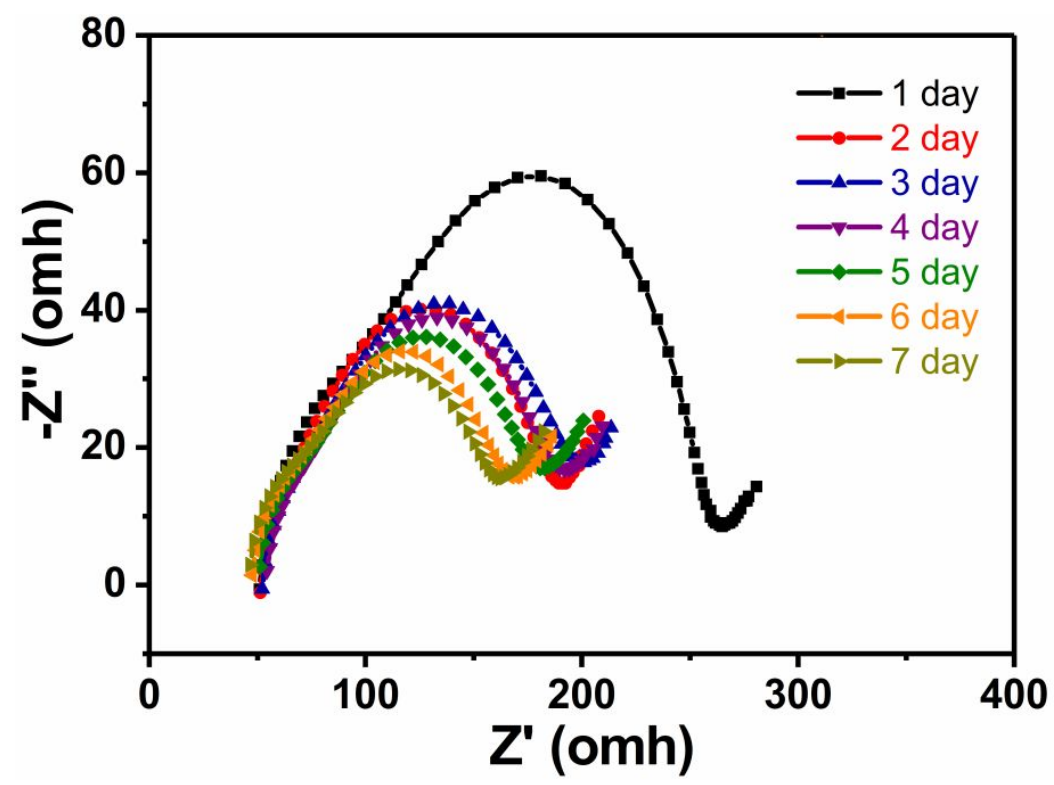

Figure S19. Impedance spectra of the $\mathrm{Li}\left|\mathrm{PEGDA}_{600} \mathrm{~S}-\mathrm{POSS}\right| \mathrm{Li}$ symmetrical cell at $60^{\circ} \mathrm{C}$ with different storage time. 